\title{
Analyse taphonomique et spatiale de palimpsestes d'occupations moustériennes de l'abri inférieur du Moustier (Dordogne, France)
}

A Taphonomic and Spatial Analysis of Mousterian Palimpsests at Le Moustier (Dordogne, France)

Marc Thomas, Emmanuel Discamps, Brad Gravina et Jean-Pierre Texier

\section{OpenEdition \\ Journals}

Édition électronique

URL : http://journals.openedition.org/paleo/4897

DOI : $10.4000 /$ paleo.4897

ISSN : 2101-0420

Éditeur

SAMRA

Édition imprimée

Date de publication : 30 décembre 2019

Pagination : 278-299

ISSN : $1145-3370$

Référence électronique

Marc Thomas, Emmanuel Discamps, Brad Gravina et Jean-Pierre Texier, « Analyse taphonomique et spatiale de palimpsestes d'occupations moustériennes de l'abri inférieur du Moustier (Dordogne, France) », PALEO [En ligne], 30-1 | 2019, mis en ligne le 29 mai 2020, consulté le 07 juillet 2020. URL : http://journals.openedition.org/paleo/4897 ; DOI : https://doi.org/10.4000/paleo.4897

\section{cc)}

PALEO est mis à disposition selon les termes de la licence Creative Commons Attribution - Pas d'Utilisation Commerciale - Pas de Modification 4.0 International. 
ANALYSE TAPHONOMIQUE

ET SPATIALE DE

PALIMPSESTES

D'OCCUPATIONS

MOUSTÉRIENNES

DE L'ABRI INFÉRIEUR

DU MOUSTIER

(DORDOGNE, FRANCE)

\author{
Marc Thomas \\ Emmanuel Discampsa, \\ Brad Gravinab, \\ Jean-Pierre Texier ${ }^{b}$
}

a. TRACES, UMR 5608 - Université Toulouse Jean Jaurès, 5 Allée Antonio Machado, FR-31058 Toulouse Cedex 9 - marcthomas1@hotmail.fr ; ediscamps@gmail.com

b. PACEA, UMR 5199 - Université de Bordeaux, Bât. B18, Allée Geoffroy SaintHilaire CS 50023, FR-33615 Pessac Cedex - gravina.brad@gmail.com ; j-pierre.texier@wanadoo.fr

PALEO 30 | t. 1

DÉCEMBRE 2019

PAGES 278 À 299

MOTS-CLÉS Densité des vestiges lithiques et osseux, processus de formation des sites, taphonomie, analyse spatiale, Moustérien, Le Moustier.
Un des enjeux majeurs de l'archéologie paléolithique reste la compréhension des processus, naturels comme anthropiques, qui participent à la formation de palimpsestes archéologiques. Un bon nombre de sites du Paléolithique moyen français se caractérise par de tels palimpsestes, constitués d'importantes accumulations de vestiges lithiques et fauniques, dont les variations de densité et de proportions relatives sont souvent difficiles à interpréter. Cet article a pour objectif d'apporter des éléments de discussion concernant l'interprétation des variations de densité en vestiges archéologiques et des proportions inter-matériaux à l'intérieur de certains des niveaux archéologiques identifiés lors de la reprise des fouilles à l'abri inférieur du Moustier. Une analyse spatiale et taphonomique a donc été mise en œuvre afin d'accéder aux modalités de la formation de ces nappes de vestiges. Les résultats de l'étude géo-archéologique ont justifié le choix d'un certain nombre d'outils analytiques : calculs de densité en matériel archéologique et des proportions entre matériaux, granulométrie des vestiges lithiques, analyse de la fabrique des vestiges, étude des états de surface du matériel lithique et analyse spatiale des raccords. Les résultats obtenus montrent que l'organisation et la composition finale des nappes de vestiges sont inféodées aux processus géologiques qui sont intervenus dans le site, i.e. processus fluviatiles, ruissellement et éboulisation des parois de l'abri. Cependant, la composition des ensembles lithiques ne semble pas avoir été significativement modifiée par les processus de formation du site, en autorisant ainsi une analyse technoéconomique. En revanche, la composition des ensembles fauniques (notamment les proportions os brûlés/non brûlés et os compact/os spongieux) pourrait avoir été affectée. 
A Taphonomic and Spatial Analysis of Mousterian

Palimpsests at Le Moustier (Dordogne, France).

One of the major concerns of Palaeolithic archaeology is understanding the various natural and anthropogenic processes underlying the formation of archaeological palimpsests. Many Middle Palaeolithic sites comprise rich accumulations of lithic and faunal remains. Here we explore variations in artifact types and inter-material densities encountered during new excavations at the lower shelter of Le Moustier. To this end, we mobilise spatial and taphonomic data to assess the multiple depositional and post-depositional processes responsible for the composition and organization of archaeological material. A combination of geoarchaeological information with density calculations, particle size distribution of lithic objects, fabric analysis, surface conditions of lithic artefacts and the statistical analysis of refitted lithic artefacts suggests the organization and ultimate composition of the remains to be dependent on natural formation processes, including flooding, surface water runoff and the desquamation of the shelter walls. While the overall homogeneity and technological nature of the lithic industries appears unaffected by such processes, the same cannot be said for the faunal material.

\section{INTRODUCTION}

En archéologie paléolithique, la quantité et la densité de matériel retrouvés à la fouille sont souvent utilisés pour discuter de l'intensité des occupations (e.g. Histock 1981; Pargeter et al. 2018) ou pour caractériser la fonction du site (e.g. Brugal 2006 ; Rendu et al. 2011). Pourtant, nombre de processus naturels peuvent modifier l'organisation et la composition de nappes de vestiges archéologiques (Texier 2000). La compréhension des processus, naturels comme anthropiques, qui participent à la formation de palimpsestes archéologiques est donc un prérequis nécessaire à toute interprétation.

La reprise des travaux de terrain à l'abri inférieur du Moustier a mis en évidence des niveaux très riches en matériel lithique associés à des proportions variables de matériel faunique, organisées en nappes de vestiges montrant des répartitions spatiales inégales. C'est principalement sur la base de ces différences de densité que trois ensembles archéo-stratigraphiques ont été distingués dans la zone nouvellement fouillée (zone B ; fig. 1 et 2). Leur étude fait l'objet de cet article. Dans le cadre de ce dernier, ces ensembles sont temporairement numérotés de 1 à 3 du sommet à la base, en attendant la fin des fouilles dans les différents secteurs du site et la définition d'un découpage stratigraphique définitif. L'extrême base de la couche H n'a pas encore été atteinte.

Deux grands types de facteurs, susceptibles d'interagir, peuvent être responsables des variations identifiées dans ces ensembles: le facteur anthropique et le facteur naturel. L'importance du premier a été testé lors d'une étude techno-économique menée sur le matériel lithique issu des ensembles 1 et 2 évoqués plus haut (Thomas et Gravina, ce volume). Cette approche a montré leur forte homogénéité. Leur formation ne peut donc pas être imputée à des changements dans la fonction ou dans le fonctionnement du site. Pour évaluer l'influence du second facteur, i.e. l'impact des processus dépositionnels et post-dépositionnels, nous proposons une étude taphonomique combinée à une analyse de la répartition spatiale des différents types de vestiges.

\section{1 | PRÉSENTATION DU SITE, CORPUS ET MÉTHODE DE FOUILLE}

Composé de deux abris superposés, le gisement du Moustier (Dordogne, France) est un lieu clef dans la reconnaissance et la caractérisation du Moustérien depuis plus de 150 ans (Lartet et Christy 1865-1875 ; Mortillet (de) 1869 ; Peyrony 1930 ; Bordes 1948 ; Laville et Rigaud 1973 ; Valladas et al. 1986). Malgré son importance, notre compréhension de la mise en place des dépôts archéologiques et de leur éventuelle réorganisation par des processus post-dépositionels reste très partielle. Fouillé principalement par D. Peyrony avant la Première Guerre mondiale (Peyrony 1930), des travaux récents sur les collections du Moustier ont depuis remis en cause la validité de la séquence archéologique originellement décrite (Gravina et Discamps 2015 ; Gravina 2017; Discamps et Lemeur, ce volume) et justifié la reprise des opérations de terrain (Gravina, Discamps et al. 2014, 2015, 2016, 2017, 2018). Ces nouvelles fouilles se concentrent sur deux 


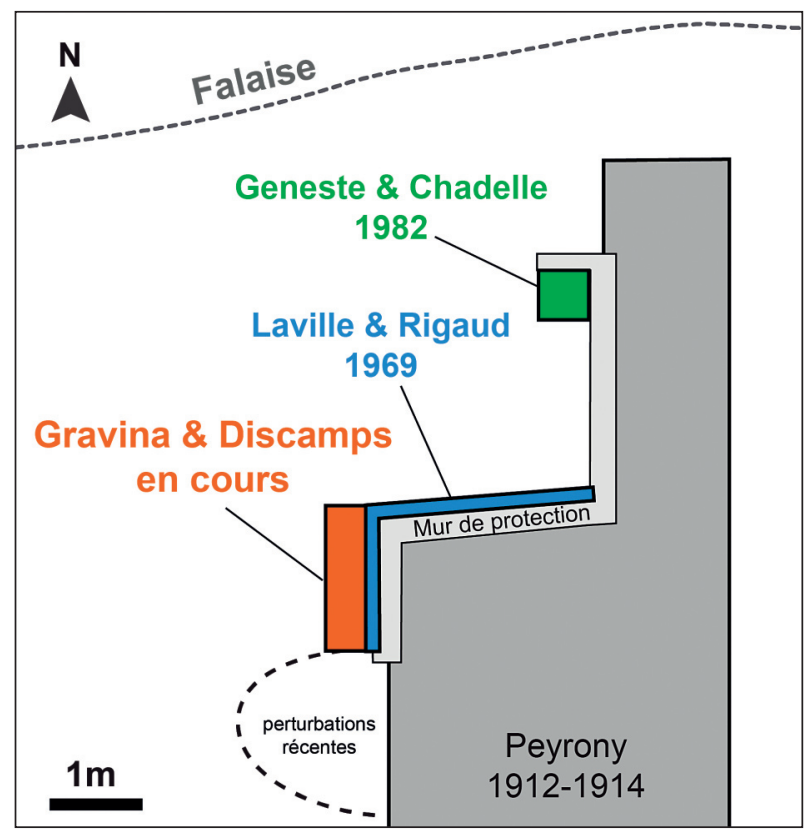

zones, dénommées $B$ et $A$, qui préservent des dépôts correspondant, respectivement, aux couches $\mathrm{G}$ à $\mathrm{H}$ et I à $L$ de D. Peyrony (1930). Les dépôts fouillés dans la zone B sont corrélés de façon fiable avec le sommet de la couche H (Discamps et al. 2016), datée de la première moitié du MIS 3 (Valladas et al. 1986). Initialement attribuées au Moustérien de Tradition Acheuléenne de type B (Bordes 1948 ; Soressi 2002), les industries lithiques de la couche $\mathrm{H}$ appartiennent au techno-complexe Discoïde (Gravina et Discamps 2015 ; Thomas et Gravina, ce volume) et sont associés à une faune dominée par les Bovinés et le Cerf (Discamps et Lemeur, ce volume).

La zone B correspond à trois quarts de mètres carrés alignés (C50a, C49c, C49a) qui font l'objet de fouilles depuis 2015.

Des décapages de $2 \mathrm{~cm}$ d'épaisseur sont effectués par sous-carré de $50 \mathrm{~cm}$ de côté, tous les vestiges archéologiques de plus de $2 \mathrm{~cm}$ sont cotés à l'aide d'un théodolite et les sédiments issus de chaque décapage sont systématiquement tamisés à l'eau selon des mailles successives de $4 \mathrm{~mm}$ et 1,6 $\mathrm{mm}$. Lors de la réalisation des décapages, les blocs supérieurs à dix centimètres sont directement écartés du seau du fouilleur. Le litrage, i.e. le volume de sédiment contenu dans le seau, est noté à la fin de chaque décapage. Lorsque les vestiges sont au moins deux fois plus longs que larges, une mesure de la fabrique (orientation et pendage) est réalisée avec un inclinomètre numérique. Lors de la fouille, lorsqu'un changement de sédiment est constaté, un nouveau décapage est entamé au sein d'une nouvelle unité stratigraphique de terrain. Ici, il n'est fait mention ni des décapages ni des US de terrain, le matériel archéologique étant redistribué au sein d'ensembles a posteriori lors de la confrontation des observations de terrain aux études du matériel lithique, du matériel faunique et suite aux analyses spatiales et taphonomiques (Discamps et al. 2019). C'est principalement sur la base de différences de densités en vestiges que trois ensembles ont été distingués (fig. 2 ; tabl. 1). Cette démarche entend différencier les découpages d'ordre géologique réalisés sur le terrain (lithostratigraphie) des ensembles définis

\section{FIGURE 1}

Emprise des différentes opérations de terrain dans l'abri inférieur du Moustier pour les dépôts correspondant aux couches $\mathrm{G}$ et $\mathrm{H}$ de Peyrony. Le bloc orange correspond à la « Zone B » des nouvelles fouilles.

Location of the different excavations of Peyrony's layers $G$ and $H$ in the lower shelter of Le Moustier. The orange square corresponds to «Zone $B$ » of the new excavations.

par des analyses d'ordre strictement archéologique (archéostratigraphie). Les refus de tamis sont attribués à l'un des ensembles archéostratigraphiques lorsque la proportion de pièces cotées appartenant au décapage appartient également à au moins $75 \%$ à un ensemble (tab.1). Les refus de tamis issus de décapages à cheval sur deux ensembles (moins de 75 \% du matériel coté appartient à la fois à ce décapage et à un unique ensemble) sont traités séparément lorsqu'il est fait mention de l'étude du matériel issu des refus de tamis.

\section{2 | DONNÉES GÉO-ARCHÉOLOGIQUES}

Dans l'abri, l'accrétion sédimentaire résulte de phénomènes d'inondation périodiques alternant avec des processus de ruissellement et d'éboulisation (Texier, in Gravina, Discanps et al. 2017, 2018). Ces mécanismes sont à l'origine de la mise en place des couches $G$ à $K$ de Peyrony, datées entre environ 56 et $40 \mathrm{ka}$ cal. BP (Valladas et al. 1986), c'est-à-dire de la première moitié du MIS 3. Les nouvelles données géomorphologiques, minéralogiques et sédimentologiques recueillies montrent que les dépôts des secteurs fouillés actuellement sont organisés en séquences répétitives comportant deux termes (Texier op. cit.) :

- un terme inférieur à base érosive, très riche en éléments archéologiques disposés principalement en conformité avec la stratification

- un terme supérieur massif, de type diamicton, nettement plus pauvre en artéfacts, et contenant de nombreux fragments calcaires plus ou moins émoussés

Le terme inférieur a été interprété comme le témoignage de brefs épisodes d'inondation de la Vézère tandis que le terme supérieur représenterait un laps de temps beaucoup plus long au cours duquel se sont manifestés des phénomènes de ruissellement et d'éboulisation (Texier op. cit.) 

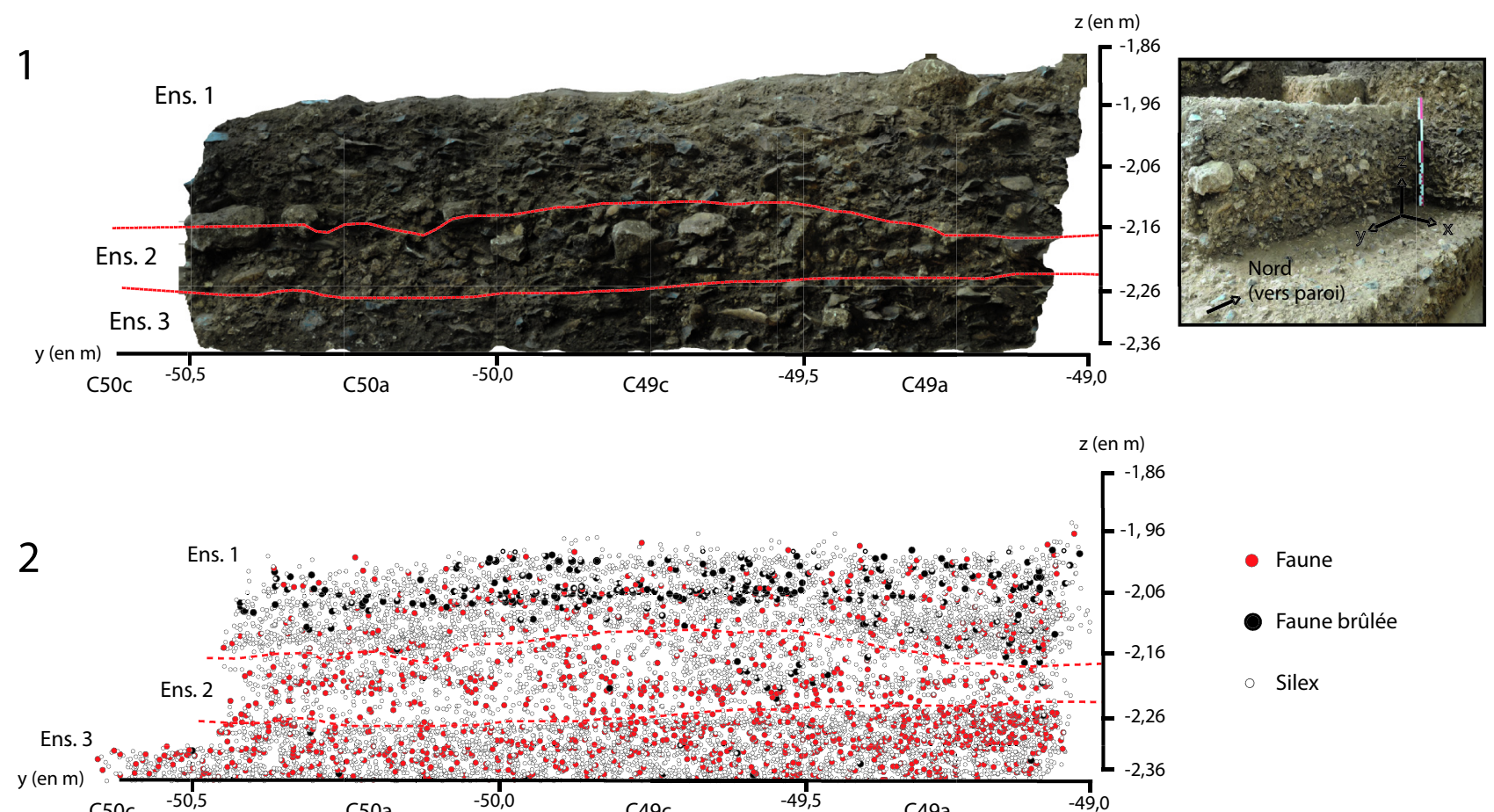

C50c

$\mathrm{C} 50 \mathrm{a}$

$\mathrm{C} 49 \mathrm{c}$

C49a

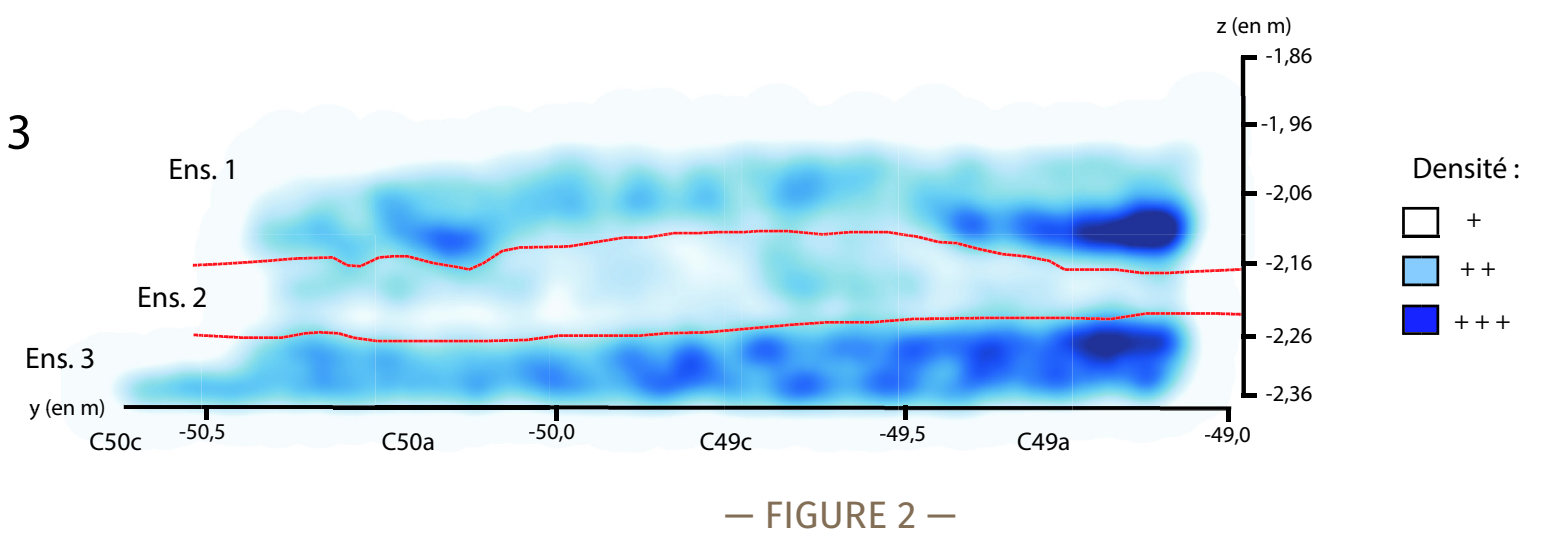

Définition des ensembles d'étude : 1: orthophotographie de la coupe sagittale de la zone $\mathrm{B}$ avec délimitation des différents ensembles étudiés, 2 : Projection sagittale des vestiges lithiques, fauniques et fauniques brûlés supérieurs à $2 \mathrm{~cm}$ cotés lors de la fouille, 3 : Carte de la différence de densité des vestiges cotés.

D'après les diagrammes de projections des pièces lithiques et osseuses, la distribution des artéfacts concorde parfaitement avec l'organisation sédimentaire décrite ci-dessus: le terme inférieur des séquences est très riche en silex tandis que leur nombre diminue progressivement de la base au sommet du terme supérieur. Une telle coïncidence incite à penser 1) que les nappes de vestiges associées au terme inférieur des séquences résultent d'une redistribution par un phénomène naturel (ce qui n'exclut pas une certaine homogénéité) et, 2) que les vestiges contenus dans le terme supérieur proviennent d'un remaniement du niveau sous-jacent. Une telle distribution a été mise en évidence en contexte de ruissellement actuel (Lenoble 2005) et reconnue en milieu paléolithique (Lenoble et Texier 2016).

Les ensembles archéostratigraphiques 1, 2 et 3 correspondent à une alternance de ces deux termes : les ensembles 1 et 3 (plus denses) correspondent à des termes
Definition of the ensembles : 1: orthophoto of the zone B sagittal profile with the limits between each ensemble, 2: Sagittal projection of lithic artefacts, bone, and burnt bone greater than $2 \mathrm{~cm}, 3$ : Density clouds of piece-plotted artefacts. 


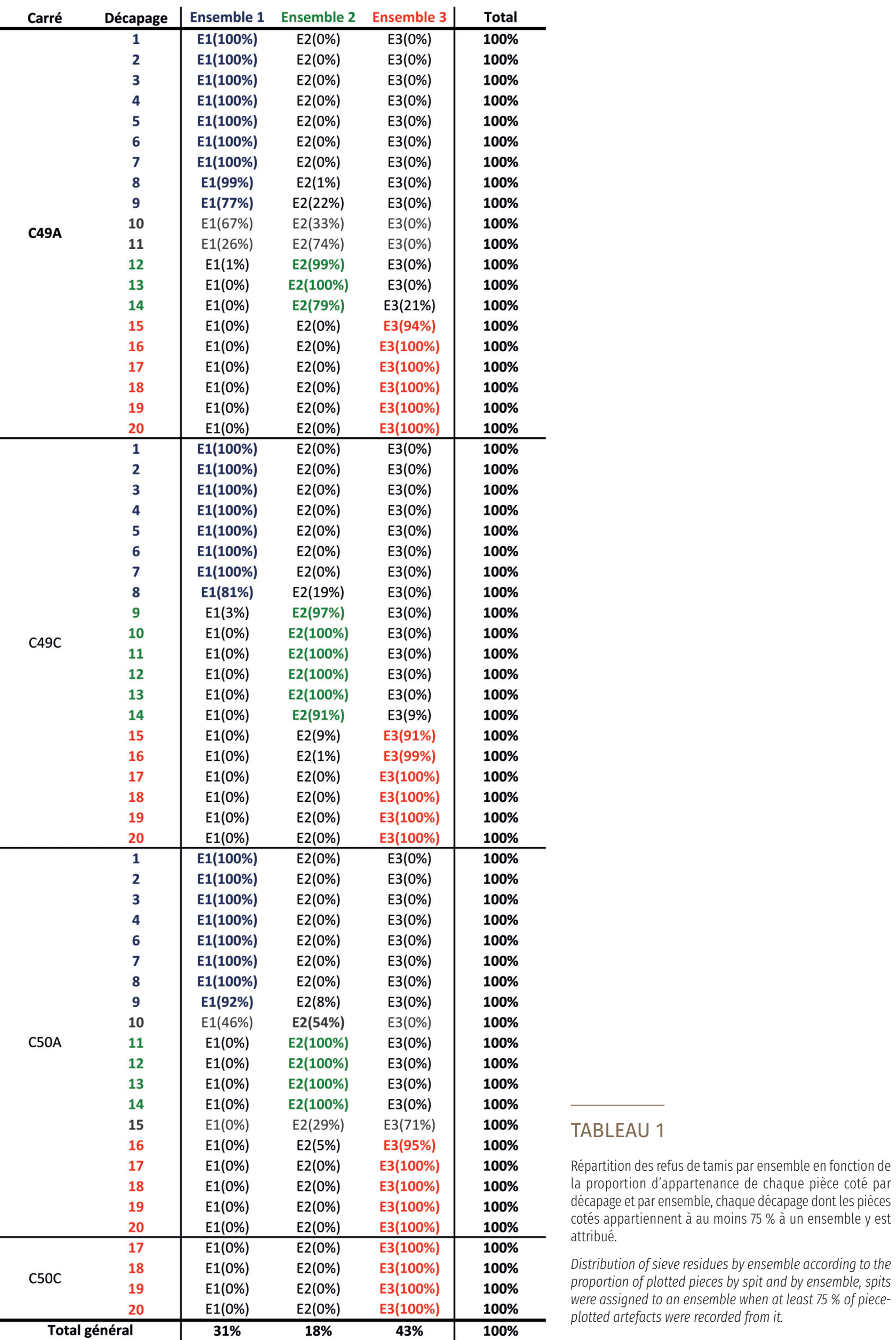




\section{3 | MÉTHODES D'ANALYSE}

\section{1 | Densité générale et proportions inter-matériaux des nappes de vestiges}

Tout d'abord une description précise des nappes de vestiges a été réalisée grâce à des projections et à l'aide de mesures de densités générales et des proportions inter-matériaux portant sur les pièces cotées et sur les refus de tamis de $4 \mathrm{~mm}$. La place prise par les blocs d'effondrement en provenance des parois de l'abri n'est pas négligeable dans certains niveaux. Ce facteur peut à lui seul expliquer les différences de densités générales constatées. En revanche, les mesures de densité stricte, i.e. le nombre de pièces par unité de volume de sédiment, devraient être indépendantes de la présence de ces blocs calcaires.

\section{2 | Fabrique des vestiges et granulométrie des vestiges lithiques}

Les processus fluviatiles et le ruissellement sont susceptibles de déplacer et de transporter des particules et des objets plus ou moins volumineux disposées sur le sol lors d'épisodes de crues ou de pluies (Bravard et Petit 1997 ; Lenoble 2005). Une telle action a été constatée en contexte archéologique. Celle-ci se traduit non seulement par des déplacements de vestiges (Schick 1986) mais aussi par des phénomènes de tri granulométrique (Schick 1986 ; Lenoble 2005 ; Bertran et al. 2006, 2012) et par l'expression de fabriques spécifiques (Isaac 1967 ; Schick 1986 ; Bertran et Lenoble 2002 ; Texier 2009). Afin de mesurer l'impact des crues de la Vézère et du ruissellement sur les ensembles de vestiges, des analyses de la fabrique des objets archéologiques (lithique et faune) et de granulométrie des vestiges lithiques ont été réalisées.

Le modèle mis en place par A. Lenoble (2005) et par P. Bertran et collaborateurs $(2006,2012)$, permet la comparaison de la granulométrie d'assemblages lithiques recueillis en contexte archéologique à des référentiels expérimentaux. Au sein d'un diagramme ternaire, sont représentées les aires de répartition attendues pour des débitages lithiques archéologiques non perturbés ainsi que pour des assemblages plus ou moins redistribués. Nous avons tenté de réduire la zone de recouvrement entre ces deux aires. Dans cette optique, nous avons redessiné les contours de l'aire des débitages expérimentaux (fig.3) en ne prenant en compte que les débitages Discoïdes. Deux nouvelles aires ont été dessinées sur le diagramme ternaire: l'une représentative de la répartition de la granulométrie de dix débitages Discoïdes menés par trois tailleurs différents (Bertran et al. 2006) et la même aire à laquelle nous avons ajouté un intervalle de confiance à 95 \% (pour chaque débitage expérimental pris individuellement) selon la méthode de T. D. Weaver et collaborateurs (2011).

L'analyse de la fabrique des vestiges ( $n=601$ mesures) a été réalisée selon la méthode élaborée par P. Bertran et A. Lenoble (2002). Les séries de mesures ont été réalisées à partir des projections du matériel effectuées avec le logiciel Datadesk. Appuyé par les calculs de densité générés sous le logiciel QGIS, les zones d'accumulation sont individualisées. Il s'agit principalement de deux séries de mesures qui se situent à la base de l'ensemble 1 (fig. 2 ; fig.6). Lorsque aucune organisation spatiale remarquable ne peut être individualisée, des ensembles de mesures horizontales sont réalisées par défaut. Considérant les propriétés physiques intrinsèques des matériaux (forme, taille, densité), nous avons distingué dans ces ensembles de mesures les vestiges lithiques des vestiges fauniques. Chaque série se compose au minimum d'une quarantaine de mesures (tabl.4). L'extension horizontale maximale entre deux mesures au sein d'une seule et même série ne dépasse jamais plus d'un mètre et demi alors que l'extension verticale maximale ne dépasse jamais plus d'une douzaine de centimètres (fig. 6 et 7).

\section{3 | Analyse des états de surface du matériel lithique}

L'analyse des états de surface du matériel lithique ainsi que leur association peut nous permettre de quantifier l'action de plusieurs agents taphonomiques (e.g. ruissellement, piétinement). Deux échantillons de vestiges lithiques au sein des ensembles 1 (base de cet ensemble, $n=1752$ éclats) et 2 ( $n=807$ éclats) ont été étudiés et sont confrontés à l'aide d'une analyse factorielle. L'étude des enlèvements mécaniques sur le bord des vestiges lithiques nous permet de mesurer l'intensité du piétinement mais elle peut aussi révéler le rôle d'écoulements d'eau de haute énergie. L'action des écoulements d'eau (fluviatile ou ruissellement) peut également provoquer un émoussé et le lustre des nervures (Lenoble 2005), voire, dans certaines conditions, l'acquisition d'une patine blanche (Texier P.-J. 1981 ; Fernandes 2012 ; Caux 2018). L'une des volontés a été d'objectiver l'enregistrement des états de surface de chaque pièce à l'échelle macroscopique. Seuls les éclats sont ici pris en considération $(n=2559)$.

L'altération mécanique du bord des vestiges a été renseignée comme suit :

- nombre d'enlèvements: $0 ; 1 ; 2 ; 3$ à 5 ; plus de 5 ; fracturation périphérique ;

- position : directe, inverse, alterne;

- pourcentage du pourtour de la pièce atteint : 0-25 \% ; 25$50 \%, 50-75 \%, 75-100 \%$;

- inclinaison du bord le plus obtus atteint: 0 à $30^{\circ} ; 30$ à $60^{\circ} ; 60$ à $90^{\circ}$

Nous avons remarqué que la patine atteint les pièces selon deux stades successifs que nous avons différenciés. Ainsi, la patine recouvre tout d'abord une partie de l'éclat sous forme de plages de moindres étendues atteignant principalement les bords et les nervures (peu patiné) puis, celle-ci peut recouvrir totalement et de manière homogène l'éclat (très patiné). Aucun éclat ne présentant un état de désilicification avancé n'a été identifié pendant l'analyse.

L'émoussé des nervures et du bord des vestiges est l'état de surface le plus compliqué à objectiver lors d'une observation macroscopique. Pour ce faire chaque pièce a été comparée de manière systématique à trois éclats sélectionnés parmi les pièces analysées et jugés représentatifs des trois stades d'usure retenus. Ces stades sont les suivants: 

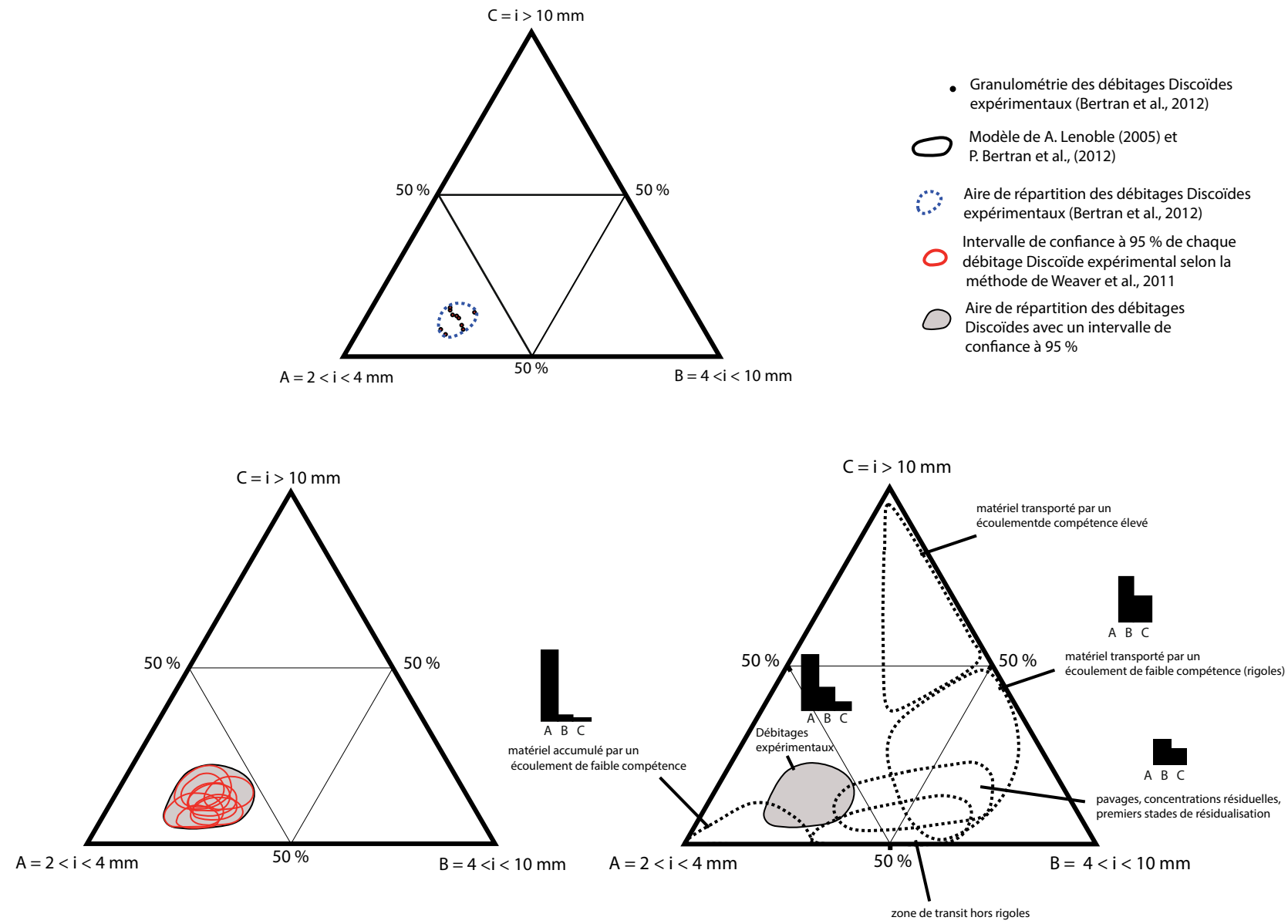

- FIGURE 3 -

Création du nouveau modèle de diagramme ternaire pour les industries Discoïde

A new ternary diagram exclusively for discoidal assemblages. exclusivement.

- non émoussé : pièce dont les nervures et les bords sont saillants

- peu émoussé : pièces qui présentent une légère usure des bords et des arêtes

- très émoussé-lustré : pièce dont les nervures et les bords sont très usées, l'émoussé peut alors s'accompagner d'un lustre.

Ces modalités d'altération sont par la suite confrontées entre elles grâce à des analyses statistiques multivariées (analyse factorielle des correspondances multiple - ACM et classification ascendante hiérarchique - $\mathrm{CAH}$ ) réalisées sous R (R Core Team, package FactoMineR cf. Lê et al. 2008).

\section{4 | Analyse des raccords}

L'analyse des raccords (dispersion verticale et horizontale, analyse statistique de l'orientation des liens) a été mise en œuvre dans le but d'évaluer l'impact des processus post-dépositionnels et l'intégrité des ensembles de vestiges (Bordes 2002, 2012). Une recherche systématique de raccords de cassures a été réalisée sur les vestiges en Sénonien blond (soit des échantillons de : 72 fragments et débris sur 163 vestiges en Sénonien blond cotés dans l'ensemble $1 ; 170$ fragments et débris sur 434 vestiges en Sénonien blond cotés dans l'ensemble 2) et plusieurs raccords de débitage et de cassure sur des matières premières singulières ont également été effectués tous matériaux confondus mais sans recherche systématique. Le nombre de raccords effectués a été limité par les contraintes de temps impartis aux travaux universitaires (travail effectué lors d'un mémoire de Master, Thomas 2017). Ainsi, l'analyse de l'orientation des liens n'a pu être mis en œuvre que dans l'ensemble 2, faute d'un nombre suffisant de liens dans les autres ensembles.

\section{4 | RÉSULTATS}

\section{1 | Description de la composition et de la densité des nappes de vestiges}

La densité générale en vestiges archéologiques est très variable selon les ensembles. Sur les projections, les ensembles 1 et 3 sont beaucoup plus denses que l'ensemble 2 (fig. 2). La place prise par les blocs dans l'ensemble 2 pourrait expliquer ce constat. Cependant, l'effectif de matériel par litre de sédiment tend à confirmer les différences de densité observées. En effet, l'ensemble 2 se compose d'un nombre de vestiges par litre de sédiment moindre (e.g. 18,7 pièces lithiques cotées par litre de sédiment) si on le compare aux ensembles 1 et 3 qui apparaissent beaucoup plus denses notamment avec un 
nombre de vestiges en silex par litre de sédiment deux fois plus important (respectivement 36,5 et 35,5 pièces lithiques cotées par litre de sédiment (tabl. 2 et 3).

La composition en matériaux des nappes de vestiges est inégale selon les ensembles. En ce qui concerne les pièces cotées, le rapport silex/faune est très élevé dans l'ensemble 1 alors qu'il tend à s'équilibrer dans les ensembles 2 et 3 (tab. 2). Alors que l'essentiel des vestiges fauniques contenu dans l'ensemble 1 sont brûlés, la quantité de ce type de vestige est très faible dans les ensembles 2 et 3 relativement aux autres catégories de vestiges. Dans les refus de tamis de faune de $4 \mathrm{~mm}$, les restes fauniques brûlés dominent très largement au sein des ensembles 1 et 2 alors que les restes fauniques non brûlés sont majoritaires dans l'ensemble 3 (fig. 4 ; tabl. 2).
Une nappe horizontale de restes fauniques brûlés apparaît sur les projections dans l'ensemble 1 (fig. 2). Sous celle-ci, des figures d'accumulation de vestiges (lithiques majoritairement), démontrant de fortes densités, sont visibles. Parmi ces dernières on note l'existence dans les ensembles 1 et 3 de structures imbriquées (en tuilage) particulièrement visibles en plan lors de la fouille. Les objets sont alors disposées à plat et se chevauchent légèrement les uns les autres. Ils forment des lignes d'artéfacts visibles en coupe (fig. 5). De telles structures sont fréquemment présentes dans les alluvions fluviatiles à galets (Doeglas 1962, Reineck et Singh 1980, Miall 1996 ; Texier 2009).

\begin{tabular}{|c|c|c|c|c|c|c|c|c|}
\hline \multirow{2}{*}{ Ensemble } & \multicolumn{3}{|c|}{ Effectifs pièces cotées } & \multicolumn{2}{|c|}{ Rapports inter-matériaux pièces cotées } & \multicolumn{2}{|c|}{ Masse refus tamis faune $4 \mathrm{~mm}$ (en g) } & \multirow{2}{*}{\begin{tabular}{|c|} 
Rapport refus de tamis faune $4 \mathrm{mn}$ \\
non brûlé/brûlé
\end{tabular}} \\
\hline & silex & faune (os+dent) & faune brûlée (os+dent) & silex/faune & faune/faune brûlée & non brûlée & brûlée & \\
\hline 1 & 3107 & 258 & 259 & 12,0 & 1,0 & 488 & 5744 & 0,1 \\
\hline 2 & 1350 & 316 & 16 & 4,3 & 19,8 & 543 & 414 & 1,3 \\
\hline 3 & 3261 & 818 & 20 & 4,0 & 40,9 & 1183 & 887 & 1,3 \\
\hline
\end{tabular}

\section{- TABLEAU 2 -}

\begin{tabular}{|c|ccc|c|}
\hline \multirow{2}{*}{ Ensemble } & \multicolumn{3}{|c|}{ Effectif/litre de sédiment } & \multirow{2}{*}{ litrage } \\
\cline { 2 - 3 } & silex & faune (os+dent) & faune brûlée (os+dent) & \\
\hline $\mathbf{1}$ & 36,5 & 2,9 & 3,3 & 77,0 \\
$\mathbf{2}$ & 18,7 & 4,5 & 0,2 & 66,0 \\
$\mathbf{3}$ & 35,5 & 8,8 & 0,2 & 89,5 \\
\hline
\end{tabular}

\section{TABLEAU 3}

Calcul de la densité stricte en matériel par litre de sédiment.

Material density calculations by sediment volume (in liters).

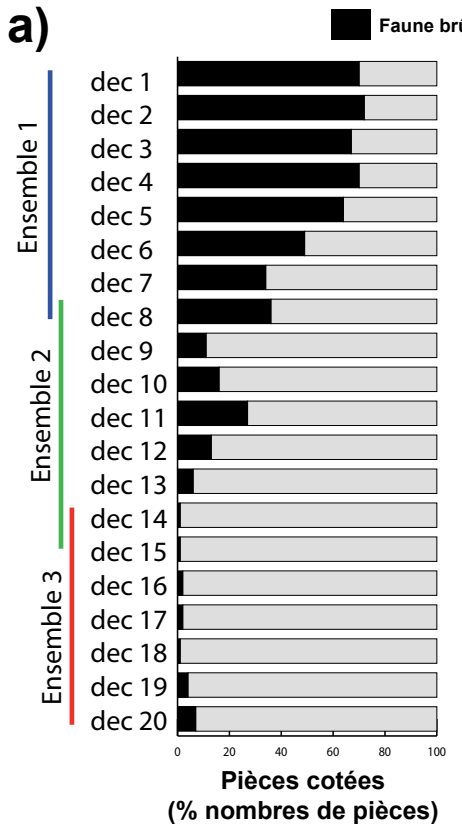

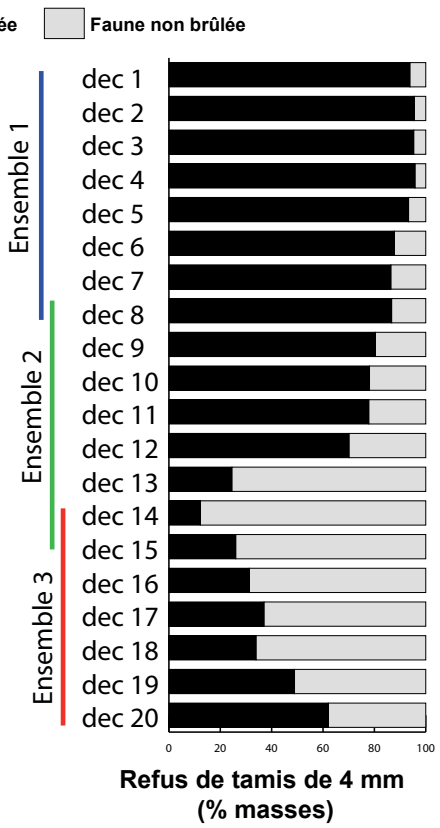

b)

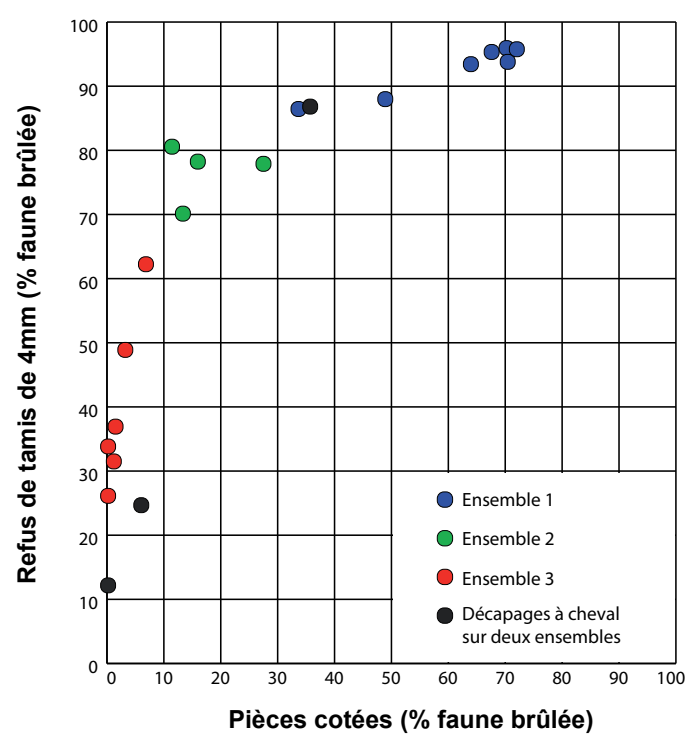

\section{- FIGURE 4 -}

A : Histogrammes cumulés de pourcentages entre faune non brûlée et brûlée par décapage parmi les pièces cotées (nombre de restes) et les refus de tamis (masse) ; B : Nuage de points du pourcentage de faune brûlée au sein des pièces cotées (en $\mathrm{x}$ ) et des refus de tamis (en Y) par décapage et par ensemble.
A: Proportions between bone and burnt bone among piece-plotted artefacts and sieve residues: $B$ : Plot of the percentage of burnt bone among piece-plotted ( $x$ axis) and sieve residues (y axis) by spit and by ensemble. 


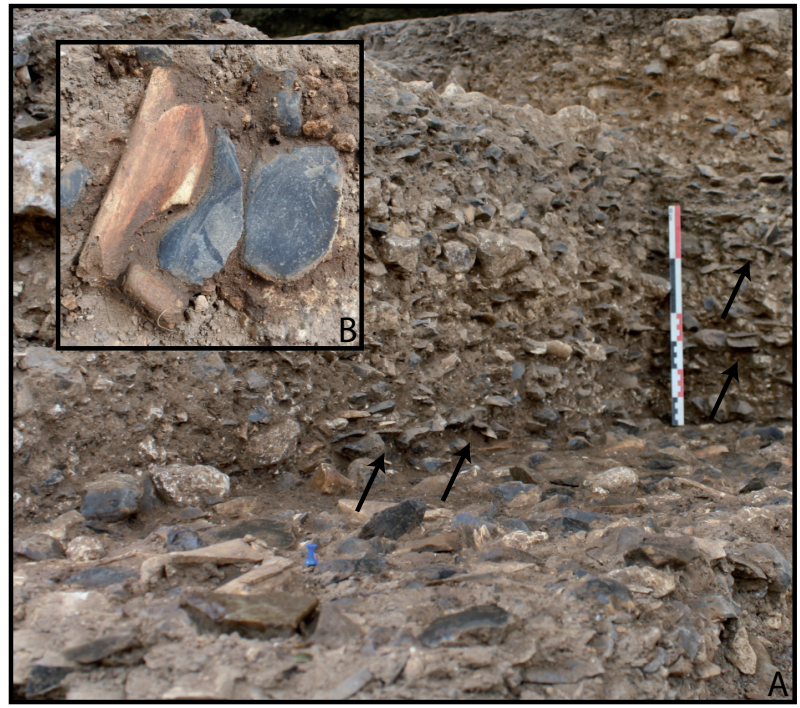

\section{2 | Analyse de la fabrique des vestiges}

Le tableau 4 et les figures 4 et 5 rapportent le résultat des analyses de fabriques. Des interprétations différentes peuvent être effectuées selon le matériau:

- Les échantillons de vestiges en silex (fig. 6) supportent l'hypothèse d'une bonne préservation.

- Les échantillons de vestiges fauniques (fig.7) possèdent quant à eux un taux d'orientation toujours supérieur aux vestiges lithiques et se situent pour la plupart en dehors de l'aire des assemblages archéologiques non perturbés. L'orientation dominante des échantillons de vestiges fauniques issus des ensembles 1 et 3 est SSO-NNE, soit, transversale à un écoulement d'eau qui serait parallèle aux parois de l'abri et conforme à la direction d'écoulement actuelle de la Vézère. Cependant, seul l'échantillon 3_1 présente un taux d'orientation statistiquement significatif ( $p=0,01$, tab. 4).

\section{3 | Granulométrie du matériel lithique}

Nous avons appliqué le modèle de A. Lenoble (2005) et P. Bertran et collaborateurs $(2006,2012)$ à tous les décapages des ensembles 1, 2 et 3 (fig. 8, tabl.5). Tous les décapages de l'ensemble 2 se situent dans l'aire des débitages expérimentaux et en dehors des aires représentatives d'assemblages redistribués lors d'expérimentations en milieu naturel (fig. 8) : il n'y a donc a priori pas de tri dimensionnel des vestiges lithiques dans cet ensemble. Le ruissellement mis en évidence par l'étude géologique n'a, selon ces résultats, pas eu d'incidence sur la composition granulométrique de cet ensemble lithique. En revanche, de nombreux décapages des ensembles 1 et 3 se situent dans des zones communes à la fois à l'aire de répartition des débitages expérimentaux et à l'aire correspondant soit à une plus forte concentration de particules fines (aire de dépôt), soit à une aire de résidualisation. Dans ces deux derniers cas, les possibles tris granulométriques mis en évidence vont dans le sens d'écoulements liquides de faible compétence, mais ces tris restent très faibles.

\section{FIGURE 5}

Disposition particulière des vestiges lithiques : A : les flèches noires soulignent des lignes d'artéfacts visibles en coupes sagittale et frontale, B : exemple de disposition en tuilage.

Particular organization of lithics: A : the black arrows highlight lines of artefacts visible in the sagittal and frontal profiles, B : example of imbricated artefacts.

\section{4 | Analyse des états de surface du matériel lithique 4.4.1 | Altération mécanique du bord des vestiges}

Une première analyse factorielle des correspondances multiples (ACM) et une classification ascendante hiérarchique (CAH) ont été réalisées afin de classer les vestiges lithiques selon les modalités renseignant l'altération mécanique de leur bord (fig. 9 et 10). L'arbre est coupé où la perte d'inertie est la moins importante pour la création des classes ( $n=5$ classes). Seul les pièces dont les bords sont altérés sont prises en considération dans l'ACM ( $n=1767$ sur un $n$ total de 2559).

Des critères à la fois d'ordre statistique (même ascendance dans l'arbre) et d'ordre archéologique nous ont poussé à regrouper certaines classes (fig. 10) :

- Alt 0 (n=792) : classe de pièces non altérées, n'apparaît pas dans l'ACM:

- Alt 1 ( $n=1151)$ : regroupement des classes 1, 2 et 3 qui correspondent à des pièces peu altérées (1, 2, 3 à 5 enlèvements voire fracturation périphérique) sur un voire deux bords (0-25\% et 25-50\%), fragiles (angles très aigus, de 0 à $30^{\circ}$ ), toujours sur l'une des deux faces seulement (position directe ou inverse);

- Alt 2 ( $n=581$ ) : classe 4 qui correspond à des pièces assez altérées (plus de 5 enlèvements) sur quasi tout leur pourtour (50 à $75 \%$ ), atteignant des bords relativement solides (30 à $60^{\circ}$ voire 60 à $90^{\circ}$ ) sur les deux faces (position alterne) ;

- Alt $\mathbf{3}$ (n=35): classe 5 qui se compose de pièces très altérées (plus de 5 enlèvements ou fracturation périphérique) sur tout leur pourtour (75-100\%), des bords solides (60 à $90^{\circ}$ ou 30 à $60^{\circ}$ ) et atteignant systématiquement les deux faces (position alterne).

Comme dans les différentes expérimentations décrites, l'altération touche en premier lieu les bords les plus fragiles des pièces. De plus, la face supérieure des objets est celle qui porte le plus souvent des enlèvements mécaniques. En considérant que la face de repos sur le sol des éclats est aléatoire lors du débitage (McPherron et al. 2014) et d'après les chiffres exprimés par Sh. McPherron et collaborateurs dans leur expérimentation (d'après ces auteurs, l'altération mécanique des bords est en moyenne 
A)
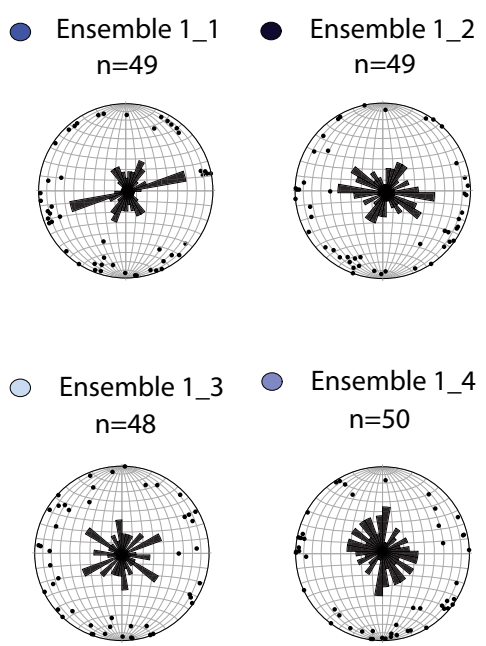

- Ensemble 2_1

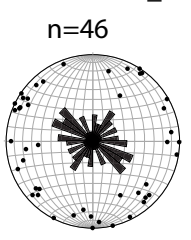

Ensemble 2_2

$\mathrm{n}=46$

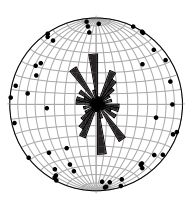

Fabrique

B)

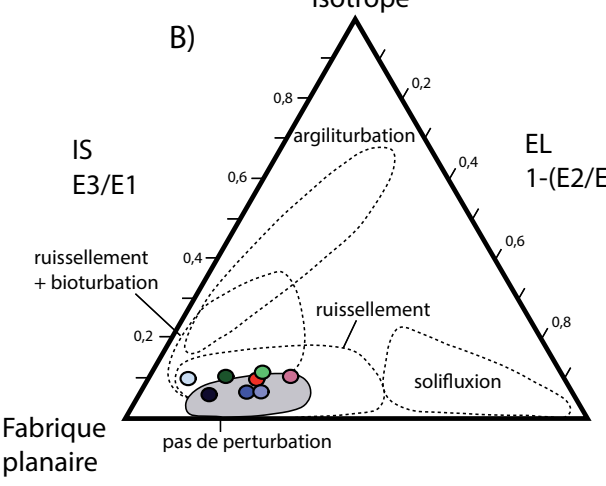

planaire
-

Ensemble 3_1

$\mathrm{n}=55$

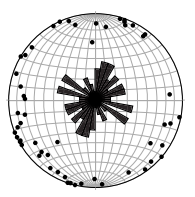

$\mathrm{n}=55$

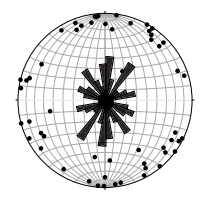

Fabrique

linéaire

Ensemble 1_1

$z$ (en $\mathrm{m}$ )

- Ensemble 1_2

Ensemble 1 3

- Ensemble 1_4

- Ensemble 2_1

Ensemble 2_2

- Ensemble 3_1

- Ensemble 3_2

C49a

\section{- FIGURE 6 -}

Résultats de l'analyse de la fabrique des vestiges lithiques. A : Diagrammes en rose des ensembles de mesure ; B : Diagramme de Benn ; C : Projection sagittale des groupes de mesures.

1,4 fois plus fréquente sur la face supérieure des éclats en silex que sur leur face inférieure) nos résultats semblent montrer une assez forte proportion d'objets dont la face supérieure est altérée ( $48 \%$ des pièces portent des enlèvements mécaniques sur leur face supérieure contre seulement 13 \% sur leur face inférieure, soit presque quatre fois plus).

\subsubsection{Analyse des correspondances multiples des états de surface lithiques}

L'association des critères relatifs aux états de surface («altération», «patine», «émoussé») tels que documentés ci-après, plus que leur analyse distincte, nous semble plus évocatrice dans l'optique de juger du degré d'homogénéité de l'assemblage et pourrait nous permettre de mieux cerner les mécanismes qui sont à leur origine (fig.9 et 11). Pour ce faire, une nouvelle analyse factorielle (avec $n=$ 2559 vestiges) et une nouvelle classification ascendante hiérarchique sont réalisées avec les nouvelles classes portant sur l'altération mécanique du bord des éclats
Fabric analysis of the lithic remains. A : Rose diagrams for the different samples; B: Benn diagram; C: Sagittal plot of the measured samples.

constituées par la première ACM et les variables «patine» et "émoussé/lustre ». Les variables «ensembles» (modalités = ensemble 1, ensemble 2), «fragmentation » (modalités = entier, fragmenté), «méthode de débitage» (modalités = Discoïde, Type Kombewa, Kombewa stricto sensu, indéterminé), "altération thermique» (modalités = brûlé, non brûlé), "longueur maximum» (modalités = classes de $5 \mathrm{~mm}$ d'intervalle) et «type matière première» (modalités = Sénonien noir, Sénonien blond, silex indéterminé, Calcédoine) sont incluses à l'ACM en tant que variables qualitatives supplémentaires.

Six classes sont suggérées par la classification ascendante hiérarchique. L'espace factoriel met en évidence une nette ségrégation de deux modalités (assez émoussé/lustré et alt 3) qui décrivent la classe 6 comportant donc des objets à la fois fortement altérés et émoussés/lustrés. Les modalités « alt 0 », « non émoussé » et «non patiné » sont proches sur l'espace factoriel et caractérisent la classe 1, soit des pièces présentant de très bons états de surfaces. Trois classes intermédiaires sont formées : d'une 


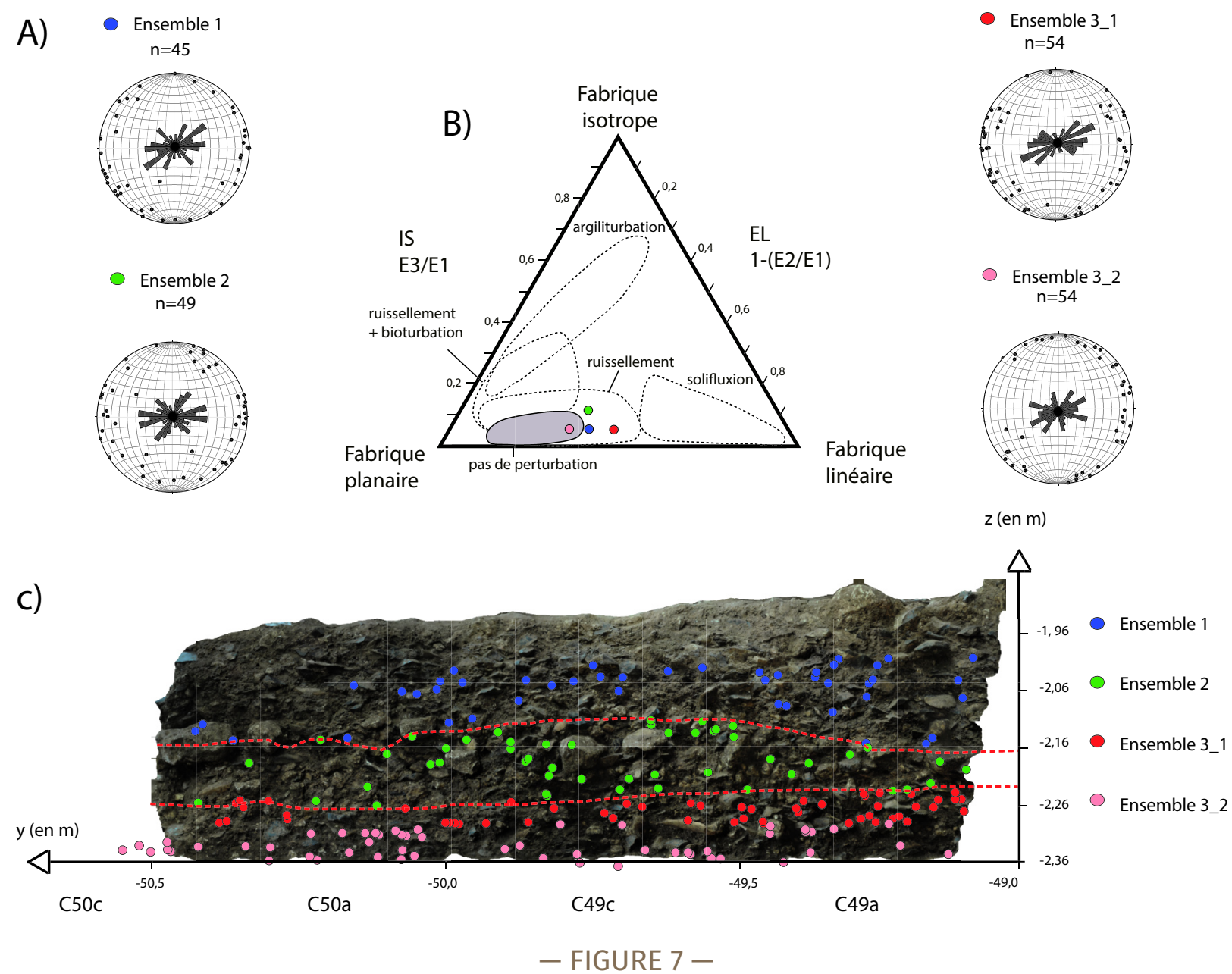

Résultats de l'analyse de la fabrique des vestiges fauniques. A : Diagrammes en

Fabric analysis of the faunal remains. A: Rose diagrams for the different samples; rose des ensembles de mesure ; B : Diagramme de Benn ; C: Projection sagittale $B$ : Benn diagram; C: Sagittal plot of the measured samples. des groupes de mesures.

\begin{tabular}{|c|c|c|c|c|c|c|c|c|c|}
\hline Matériau & Ensemble & IS & EL & E1 & E2 & E3 & L (\%) & nbr mesures & p-value \\
\hline \multirow{4}{*}{ Faune } & 1_1 & 0,06 & 0,39 & 0,60 & 0,37 & 0,04 & 23,27 & 45 & 0,08 \\
\hline & 2_1 & 0,12 & 0,36 & 0,57 & 0,36 & 0,07 & 20,39 & 49 & 0,13 \\
\hline & 3_1 & 0,06 & 0,46 & 0,63 & 0,34 & 0,04 & 30,22 & 54 & 0,01 \\
\hline & 3_2 & 0,06 & 0,33 & 0,58 & 0,39 & 0,04 & 21,15 & 54 & 0,08 \\
\hline \multirow{8}{*}{ Silex } & 1_1 & 0,07 & 0,23 & 0,54 & 0,42 & 0,04 & 13,50 & 49 & 0,40 \\
\hline & $1 \_2$ & 0,06 & 0,15 & 0,52 & 0,44 & 0,03 & 9,40 & 49 & 0,64 \\
\hline & 1_3 & 0,10 & 0,09 & 0,50 & 0,45 & 0,05 & 3,89 & 48 & 0,92 \\
\hline & 1 & 0,07 & 0,26 & 0,55 & 0,41 & 0,04 & 14,18 & 50 & 0,36 \\
\hline & 2_1 & 0,11 & 0,16 & 0,51 & 0,43 & 0,06 & 8,86 & 46 & 0,69 \\
\hline & 2 & 0,12 & 0,24 & 0,53 & 0,41 & 0,06 & 11,48 & 46 & 0,54 \\
\hline & 3_1 & 0,10 & 0,24 & 0,54 & 0,41 & 0,05 & 13,49 & 55 & 0,36 \\
\hline & 3 & 0,11 & 0,30 & 0,55 & 0,39 & 0,06 & 18,12 & 55 & 0,16 \\
\hline
\end{tabular}

- TABLEAU 4 -

Résultats de l'analyse statistique de la fabrique des vestiges (méthode Bertran Results of the fabric analysis. et Lenoble 2002).

part, les classes 2 et 3 qui se composent de pièces seulement altérées de manière plus ou mois importante et, d'autre part, la classe 4 qui comporte des pièces à la fois peu émoussées et peu patinées.

Il est tentant de corréler ces classes aux facteurs liés aux processus géologiques de formation du site et/ou aux agents post-dépositionnels, mais la forte proximité des modalités de la variable supplémentaire «ensemble» sur l'espace factoriel ne nous le permet pas. Bien que les classes objectivées par la CAH nous paraissent archéologiquement très cohérentes, cette analyse met également en évidence la forte homogénéité des états de surface au sein des ensembles: il y a, d'une manière générale, peu de pièces avec de mauvais états de surface, et ce quel que soit l'ensemble archéostratigraphique 

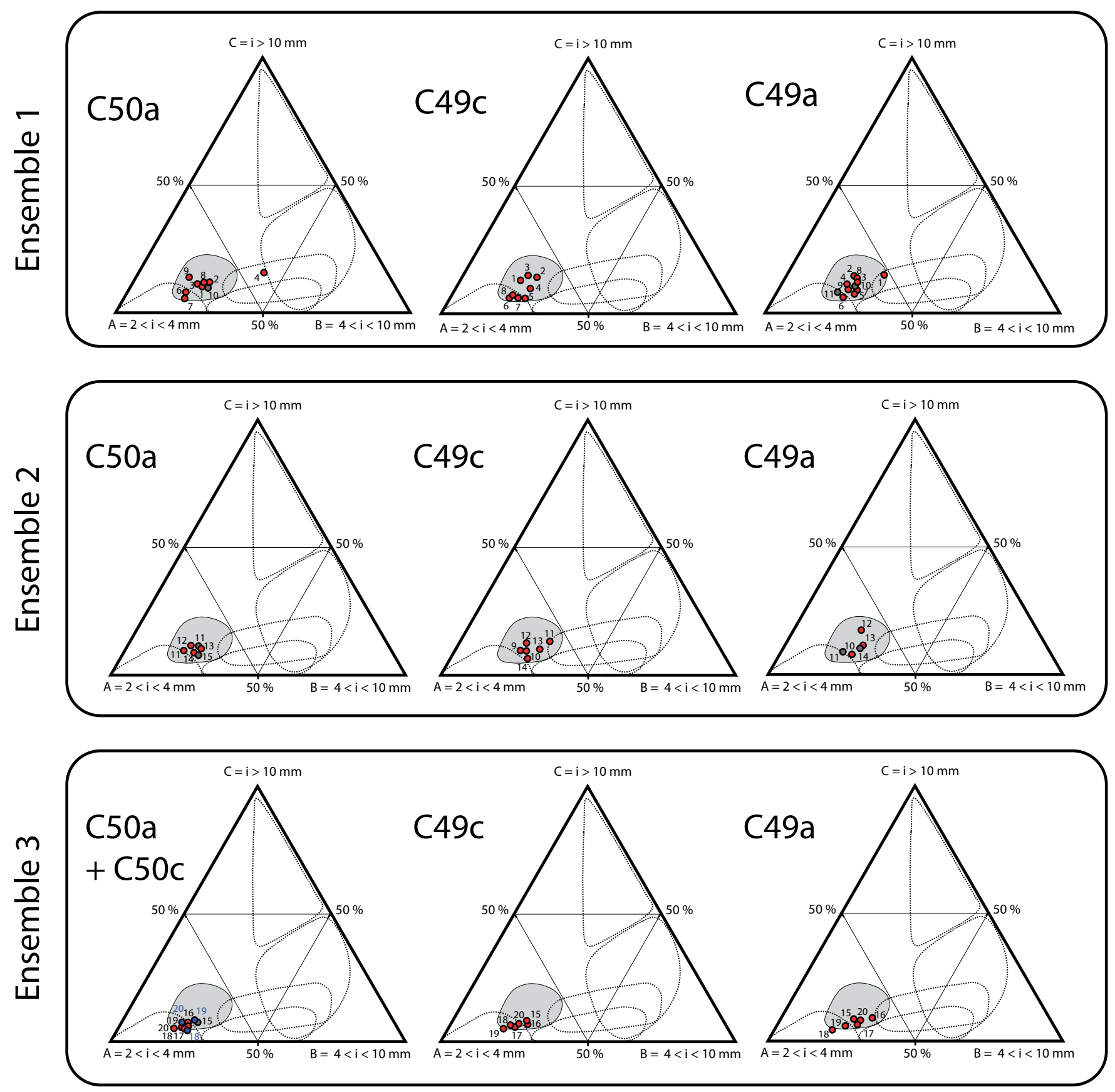

- FIGURE 8 -

Résultats de l'analyse de la granulométrie des vestiges lithiques. La zone grisée correspond à l'air de répartition de la granulométrie des débitages expérimentaux. Les zones délimitées par des pointillés noirs correspondent à des aires de répartition de la granulométrie d'ensembles expérimentaux soumis au ruissellement en milieu naturel (cf. fig. 2). Les trois décapages réalisés dans le carré C50c sont intégrés dans les résultats du carré C50a, ensemble 3.
Particle size analysis of the lithic remains. The shaded area corresponds to the distribution of experimental reduction sequences. The areas delimited by black dots correspond to the distribution ranges of the experimental reduction sequenced affected by surface water runoff (see Fig. 2). The three spits in square $\mathrm{C5Oc}$ are included with the results for ensemble 3 in square C50a. considéré. À ce stade de l'analyse, l'hypothèse la plus parcimonieuse est de considérer que les processus sédimentaires mis en jeu dans la formation de ces deux ensembles ont entraîné des états de surface similaires sur les vestiges et que les processus fluviatiles mis en jeu dans l'ensemble 1 n'ont joué qu'un rôle mineur dans leur acquisition. De la même manière, on ne peut constater de ségrégation d'aucune des variables qualitatives supplémentaires intégrées à l'ACM, les variables «altération thermique», « fragmentation », « type de matière première» et «méthode de débitage» sont très peu liées aux variables actives de l'ACM et les ellipses formées par leurs modalités se recoupent fortement (fig.12).

Il apparaît donc que :

- ni le type de matière première, ni la taille des vestiges ne sont des variables significativement actives dans l'acquisition des états de surfaces tels que discutés ici ; 


\begin{tabular}{|c|c|c|c|c|c|c|c|c|c|c|c|}
\hline arr & $2-4[$ & [4-10[ & 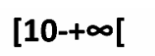 & |carre & $2-4[$ & 10 & [10-+ & car & |[2-4[ & o[ & $+\infty[$ \\
\hline C49a-1 & 159 & 102 & 43 & C49c-1 & 358 & 108 & 69 & C50a-1 & 202 & 76 & 36 \\
\hline C49a-2 & 301 & 112 & 66 & C49c-2 & 492 & 204 & 115 & C50a-2 & 343 & 140 & 64 \\
\hline C49a-3 & 297 & 119 & 55 & C49c-3 & 626 & 215 & 147 & C50a-3 & 553 & 188 & 92 \\
\hline C49a-4 & 855 & 282 & 145 & C49c-4 & 1405 & 549 & 213 & C50a-4 & 406 & 409 & 159 \\
\hline C49a-5 & 1327 & 527 & 169 & C49c-5 & 822 & 304 & 69 & C50a-5 & \multicolumn{3}{|c|}{ non existant } \\
\hline C49a-6 & 2598 & 852 & 228 & C49c-6 & 2502 & 675 & 216 & C50a-6 & 1520 & 420 & 171 \\
\hline C49a-7 & 974 & 388 & 134 & C49c-7 & 1088 & 350 & 96 & C50a-7 & 2346 & 654 & 162 \\
\hline C49a-8 & 1634 & 624 & 354 & $C 49 c-8$ & 1084 & 307 & 105 & C50a-8 & 1556 & 578 & 242 \\
\hline C49a-9 & 1747 & 605 & 237 & C49c-9 & 1375 & 442 & 205 & C50a-9 & 589 & 154 & 120 \\
\hline C49a-10 & 825 & 333 & 130 & C49c-10 & 846 & 308 & 127 & C50a-10 & 695 & 271 & 108 \\
\hline C49a-11 & 389 & 111 & 44 & C49c-11 & 224 & 119 & 55 & C50a-11 & 552 & 160 & 66 \\
\hline C49a-12 & 438 & 162 & 120 & C49c-12 & 629 & 220 & 127 & C50a-12 & 829 & 268 & 130 \\
\hline C49a-13 & 488 & 200 & 83 & C49c-13 & 655 & 303 & 112 & C50a-13 & 689 & 278 & 99 \\
\hline C49a-14 & 1577 & 553 & 159 & C49c-14 & 1711 & 664 & 176 & C50a-14 & 1429 & 527 & 164 \\
\hline C49a-15 & 1984 & 755 & 262 & C49c-15 & 1844 & 693 & 193 & C50a-15 & 1389 & 522 & 149 \\
\hline C49a-16 & 1648 & 860 & 252 & C49c-16 & 2026 & 745 & 236 & C50a-16 & 1761 & 551 & 190 \\
\hline C49a-17 & 1970 & 814 & 203 & C49c-17 & 2640 & 793 & 212 & C50a-17 & 2162 & 701 & 173 \\
\hline C49a-18 & 3628 & 956 & 214 & C49c-18 & 2610 & 706 & 231 & C50a-18 & 2299 & 714 & 162 \\
\hline C49a-19 & 2668 & 889 & 228 & C49c-19 & 3507 & 855 & 245 & C50a-19 & 2755 & 800 & 206 \\
\hline C49a-20 & 1337 & 568 & 171 & C49c-20 & 3013 & 946 & 298 & C50a-20 & 3531 & 862 & 247 \\
\hline C50c-18 & 426 & 144 & 32 & C50c-19 & 437 & 154 & 56 & C50c-20 & 765 & 228 & 61 \\
\hline
\end{tabular}

- TABLEAU 5 -

- la fragmentation des pièces archéologiques est à lier au débitage plus qu'à des facteurs d'ordre taphonomique ; - quel que soit le savoir-faire (Discoïde, Type Kombewa) mis en jeu lors du débitage, les pièces ont été soumises à des processus d'altération et d'abrasion impliquant des résultats égaux;

- une forte homogénéité de l'assemblage, quel que soit l'ensemble considéré, est mise en évidence ;

- aucune organisation spatiale remarquable des états de surface des vestiges lithiques n'est visible sur les projections (fig. 13);

- les pièces lithiques qui composent la classe 6 (moins de $3 \%$ du matériel dans les deux ensembles, fig. 11) présentent des états de surface compatibles avec un transport en milieu fluviatile, occasionnant une forte usure des bords et des nervures (émoussé, lustre, enlèvements mécaniques). Ces pièces peuvent provenir d'une partie amont du site d'où elles auraient été remobilisées et déposées à l'emplacement de la zone de fouille actuelle. Toutefois, selon les résultats des analyses technologiques du matériel lithique (Thomas et Gravina, ce volume) l'homogénéité des ensembles lithiques ne peut être remise en cause ni en termes d'économie des matières premières, ni en termes des savoir-faire et des connaissances identifiés.

\section{5 | Analyse spatiale des raccords}

L'ensemble 1 met en évidence un faible nombre de raccords (cinq uniquement, fig. 14) et ne met pas à disposition un effectif suffisamment conséquent pour tester statistiquement l'orientation des liens.

L'ensemble 2 met en évidence un nombre de raccords plus important $(n=35)$, faisant intervenir jusqu'à six pièces raccordées sur un nucléus. L'analyse statistique de l'orientation des liens $(n=35)$ de raccords de l'ensemble 2 met en évidence un taux d'orientation de $21,13 \%$, le test de Rayleigh donne une p-value de 0,20 (supérieure au seuil de significativité de 0,05 ) et permet de rejeter l'hypothèse d'une orientation préférentielle des liens de raccords dans l'ensemble 2 (fig. 15). Pour l'heure, aucun raccord ne montre une dispersion verticale qui permettrait de remettre en cause les ensembles archéostratigraphiques.

Les rapprochements de matières premières sont pour leur part assez nombreux et laissent entrevoir la possibilité de réaliser plus de raccords. Nous avons choisi de ne pas les faire figurer dans ce travail. La principale raison de ce choix tient à la relative subjectivité qu'ils soulèvent quant à leur interprétation qui se situe de part et d'autre d'une frontière fragile entre leur incertitude et les fortes conséquences en termes d'interprétations stratigraphiques qu'ils peuvent impliquer. Cette incertitude est d'autant plus grande au Moustier que nous disposons d'un corpus de matière première très homogène tout au long de la séquence. 

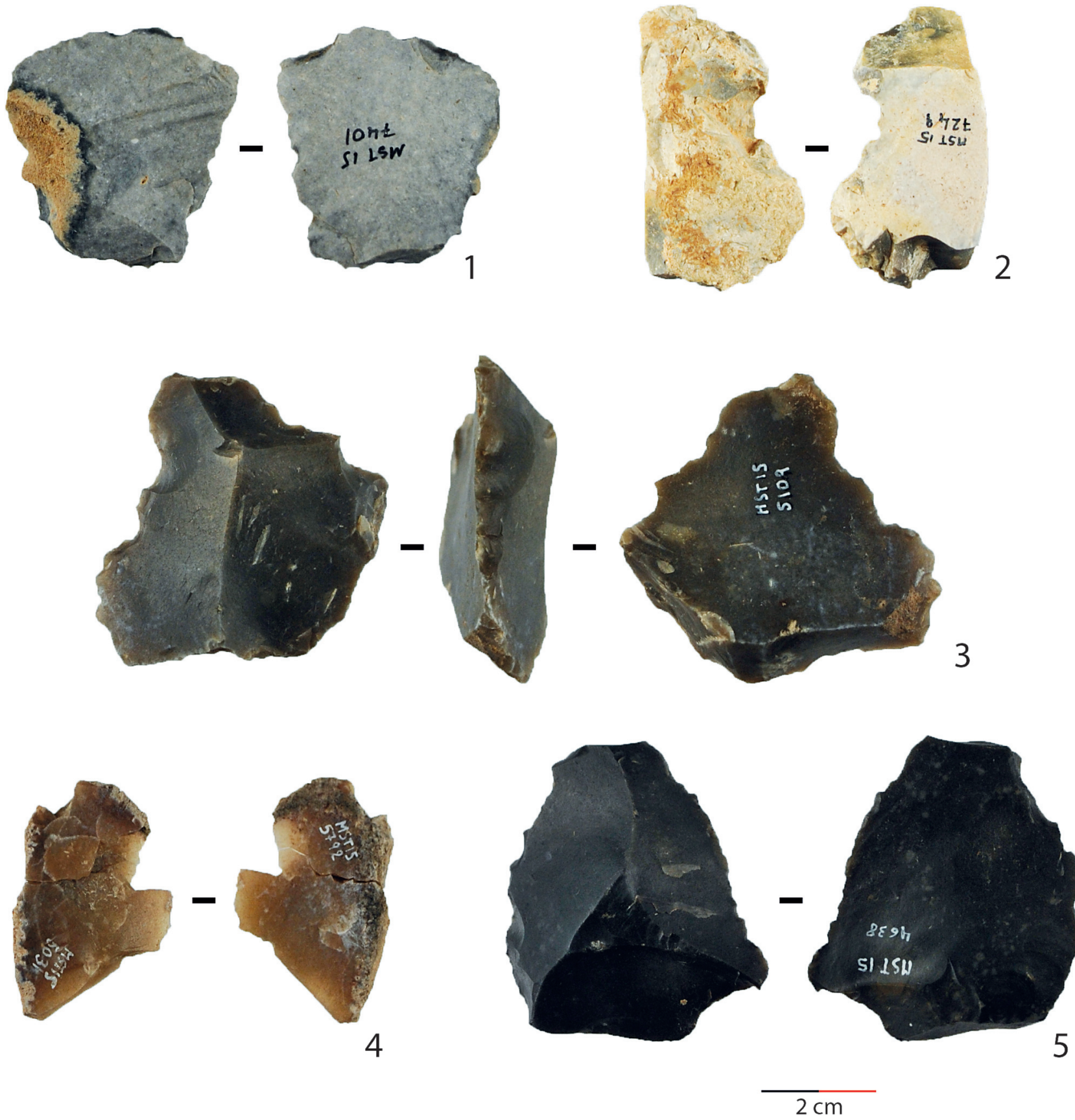

- FIGURE 9 -

Sélection de pièces évocatrices des états de surface renseignés de manière systématique à l'échelle macroscopique : 1 et 2 = pièces patinées ; 3 = pièce dont les bords sont altérés mécaniquement; 4 = pièce altérée thermiquement; 5 = pièce lustrée.

\section{5 | DISCUSSION}

Alors que de fortes perturbations engendrées par des crues de la Vézère étaient attendues dans les ensembles 1 et 3 , les résultats obtenus lors de cette étude taphonomique sont surprenants à plusieurs égards. En effet, la multiplication des outils analytiques développés convergent dans le sens d'une faible perturbation de ces ensembles. Plusieurs points méritent d'être mis en avant :
Examples of types of macroscopic surface alterations recorded for each lithic object: 1 and $2=$ patinated pieces; 3 = pieces with edge damage; $4=$ thermally altered pieces ; $5=$ lustered pieces.

\section{(1) Une faible perturbation des ensembles de vestiges lithiques}

Une faible perturbation des vestiges lithiques est mise en évidence par un tri granulométrique faible (ensembles 1 et 3) voire absent (ensemble 2), des fabriques conformes à des assemblages non redistribués et des états de surfaces globalement frais. Aucun outil employé ne permet de remettre en cause la lecture que l'on peut faire de la série lithique en termes d'économie des matières premières, des savoir-faire et des connaissances mis en œuvre. Les vestiges lithiques n'ont pas ou très peu subi de phénomènes de tris et ont été probablement simplement réorganisés quasi in situ sans recomposition importante. 


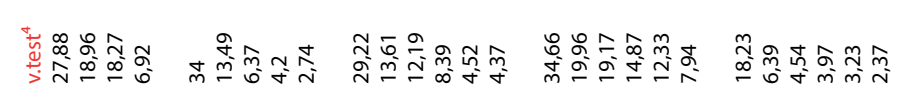

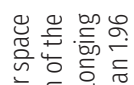

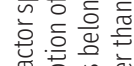

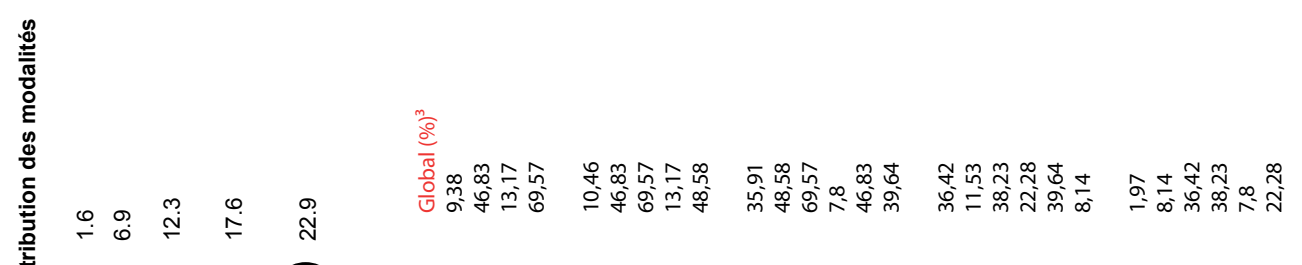

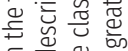

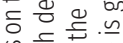

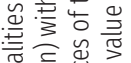

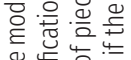

-OOOO

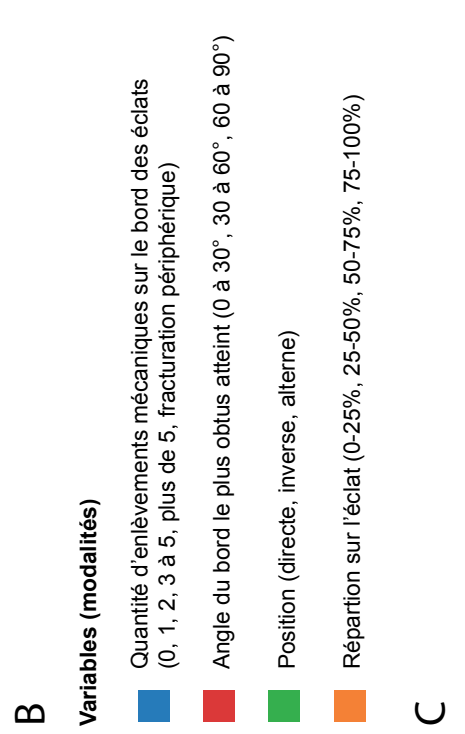

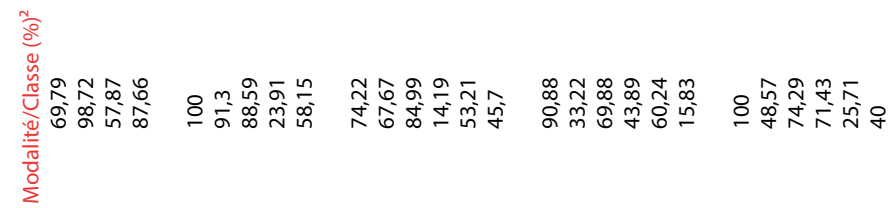

$\overline{\frac{2}{a}}$

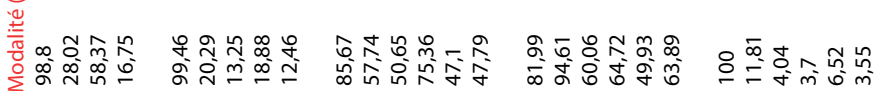

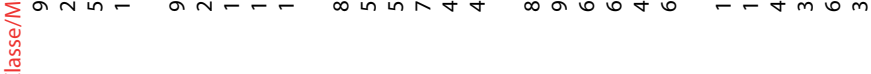

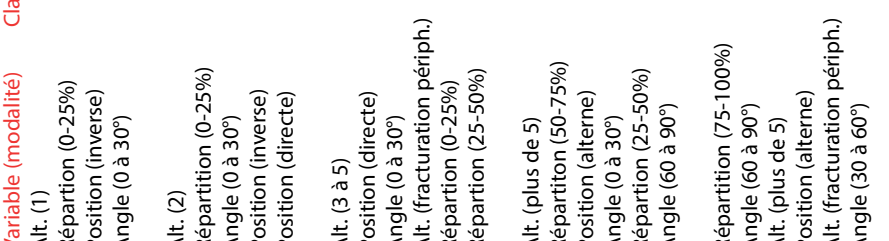

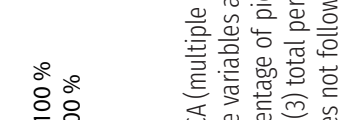

흥 정 总

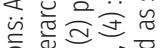

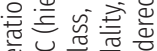

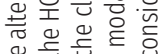

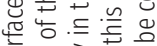

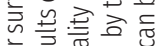

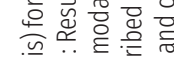

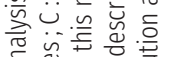

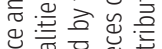

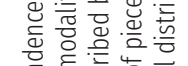

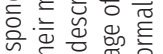

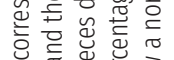

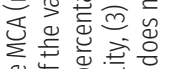

离容

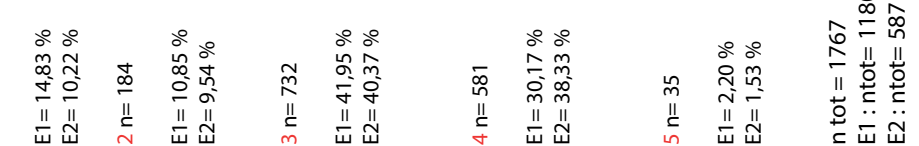

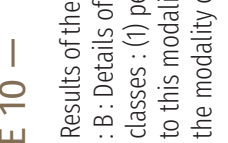

ㄸ

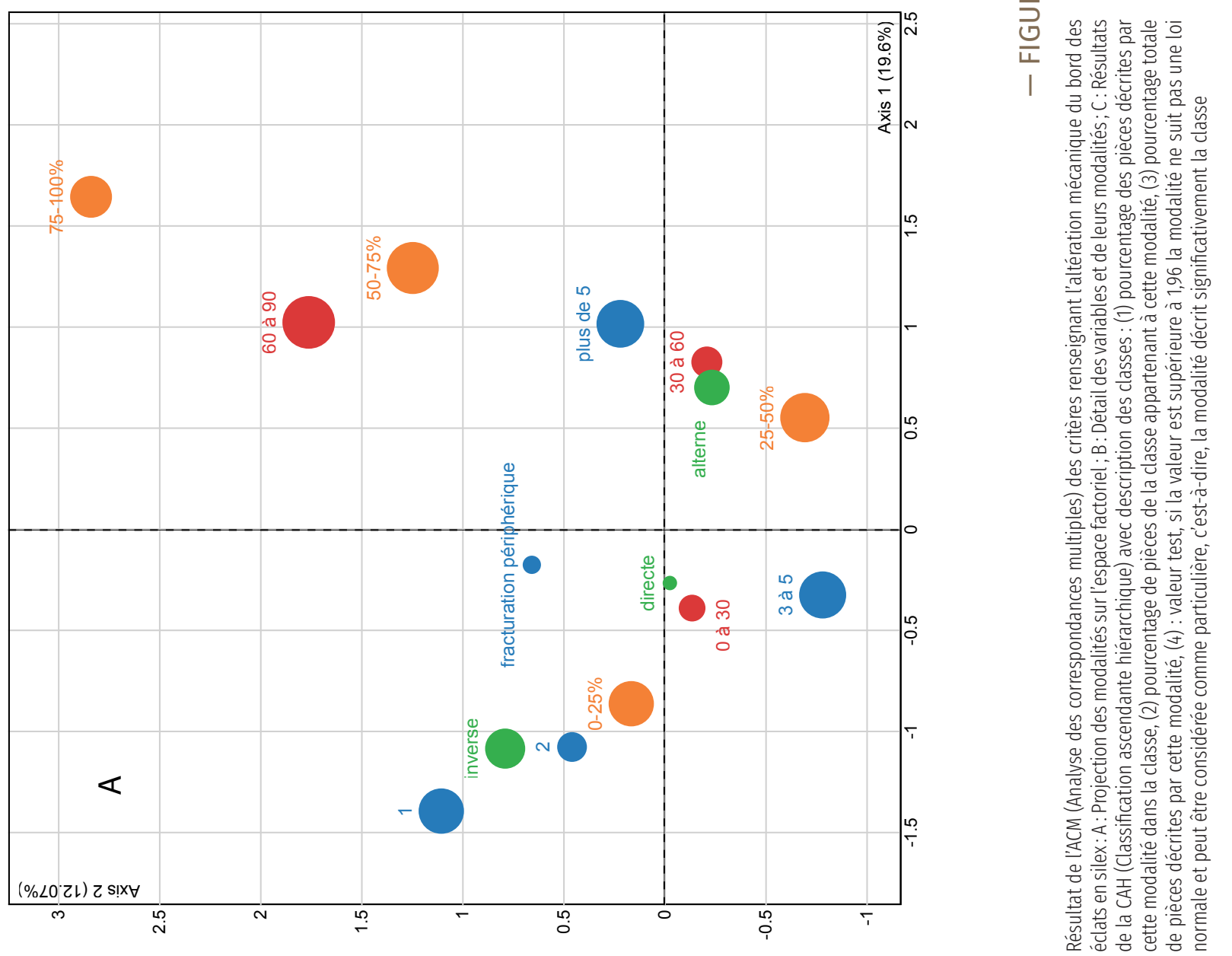




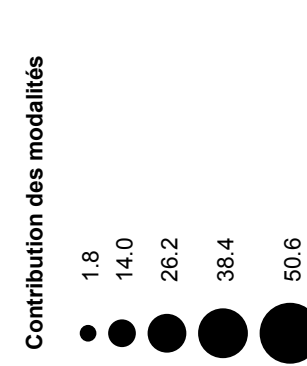

$\infty$

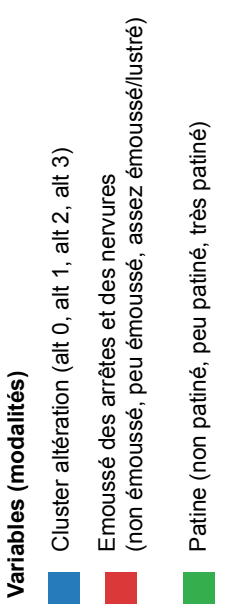

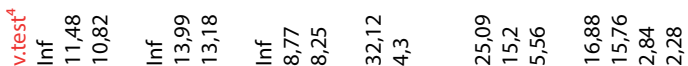
\%

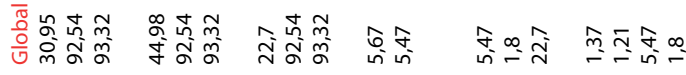
$\frac{8}{8}$

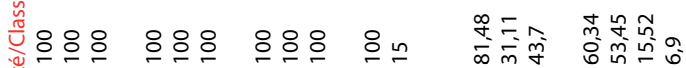

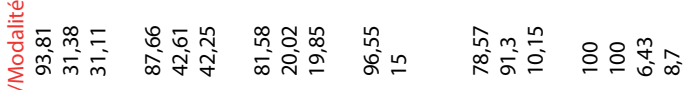

б

造 范

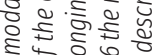

要

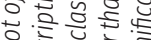

‥

证的的

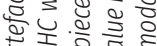

일

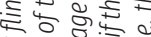

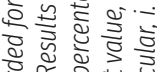

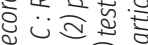

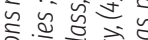

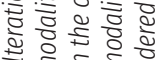

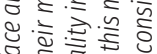

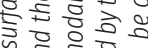

o

o

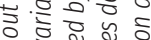

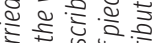

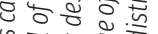

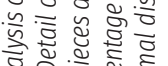

हैं क्षे

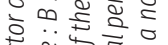

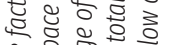

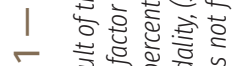

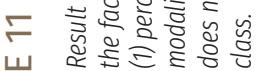

그

드. 들 总

证

웅

$\because$ 通

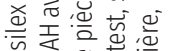

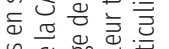

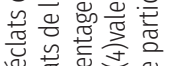

造记

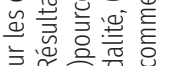

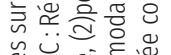

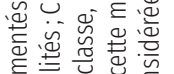

空 $\frac{\pi}{0} \frac{\pi}{0}$

过

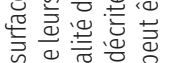

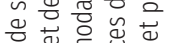

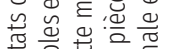

类

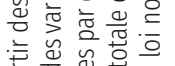

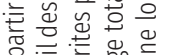

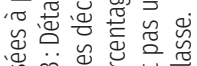

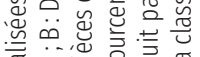

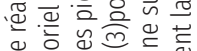

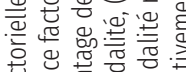

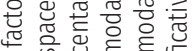

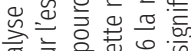

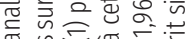

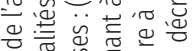

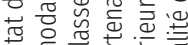

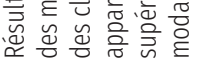




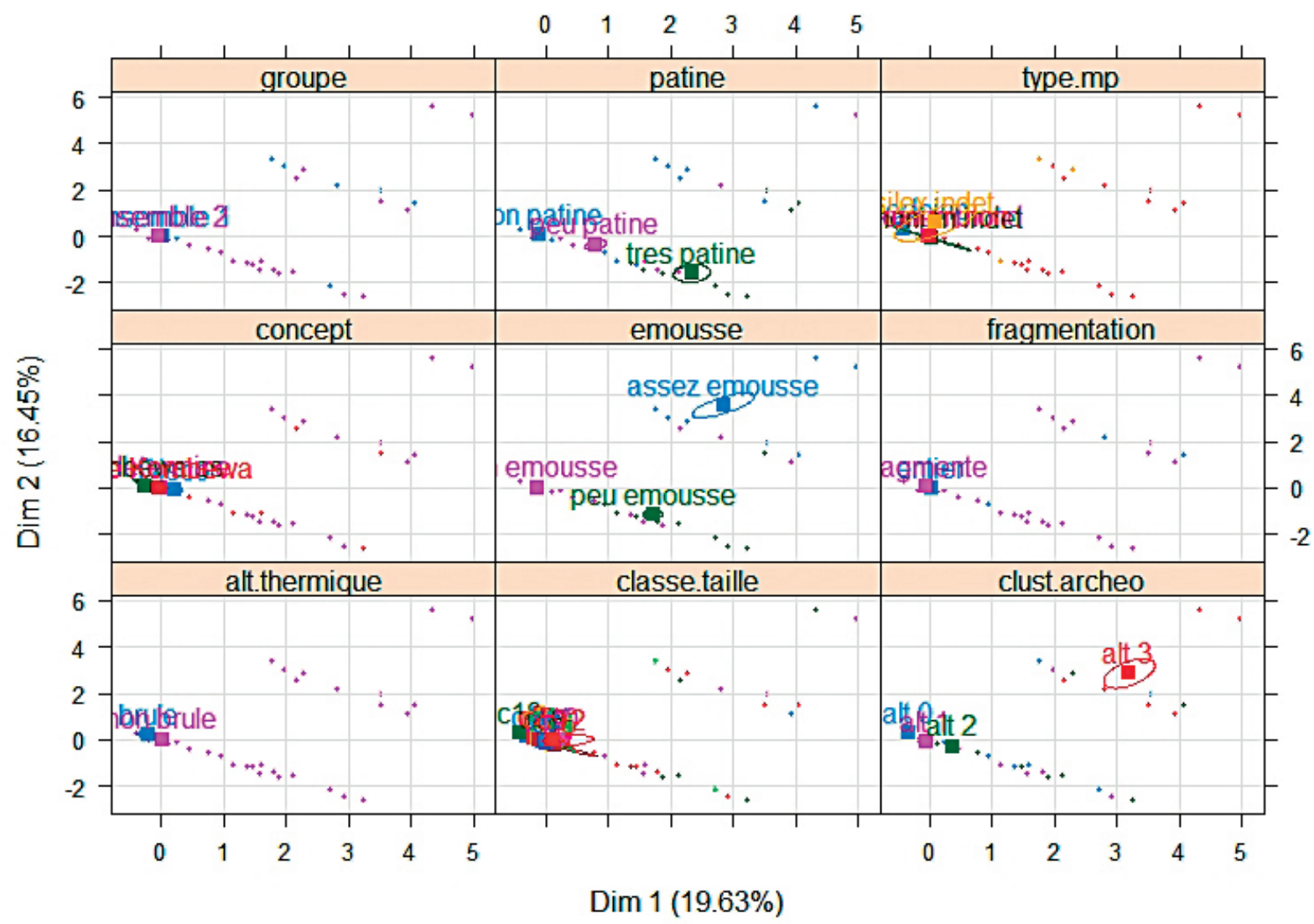

- FIGURE $12-$

Ellipses formées par les modalités des variables qualitatives supplémentaires Ellipses formed by the modalities of additional qualitative variables added to the ajoutées à l'analyse factorielle des états de surface.

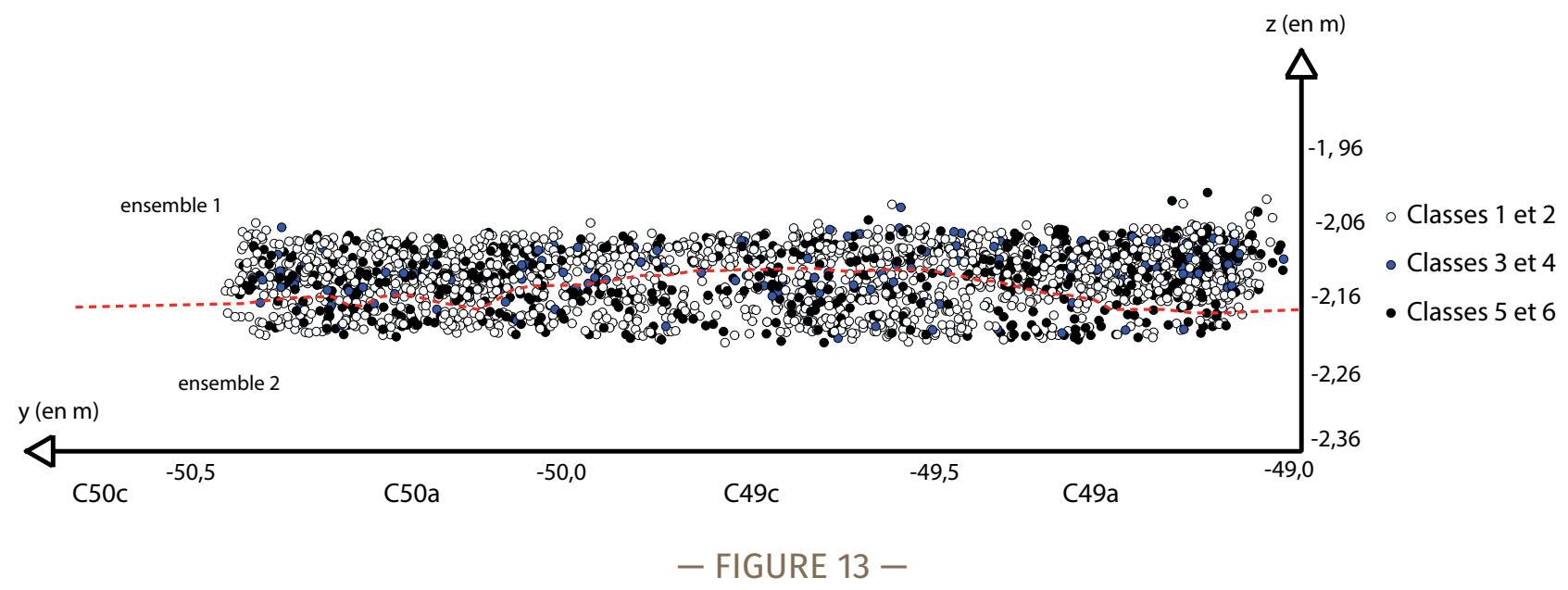

Projection des classes d'états de surface générées par la classification ascendante hiérarchique.

Plot of surface alteration classes generated by the hierarchical classification.

(2) Plusieurs arguments suggèrent un impact plus important des processus dépositionnels ou post-dépositionnels sur les vestiges fauniques

L'altération mécanique du bord des vestiges lithiques est probablement à lier au piétinement humain intervenu lors des nombreuses occupations du site. L'impact du piétinement (potentiellement combiné au weathering) a probablement eu pour effet une fragmentation d'une partie des os brûlés.

Plusieurs décapages des ensembles 1 et 3 présentent un faible tri granulométrique des vestiges en silex (accumulation de particules fines de 2 à $4 \mathrm{~mm}$ ou érosion de ces dernières). Or, les vestiges fauniques possèdent une densité moins élevée que ceux en silex et pour la 

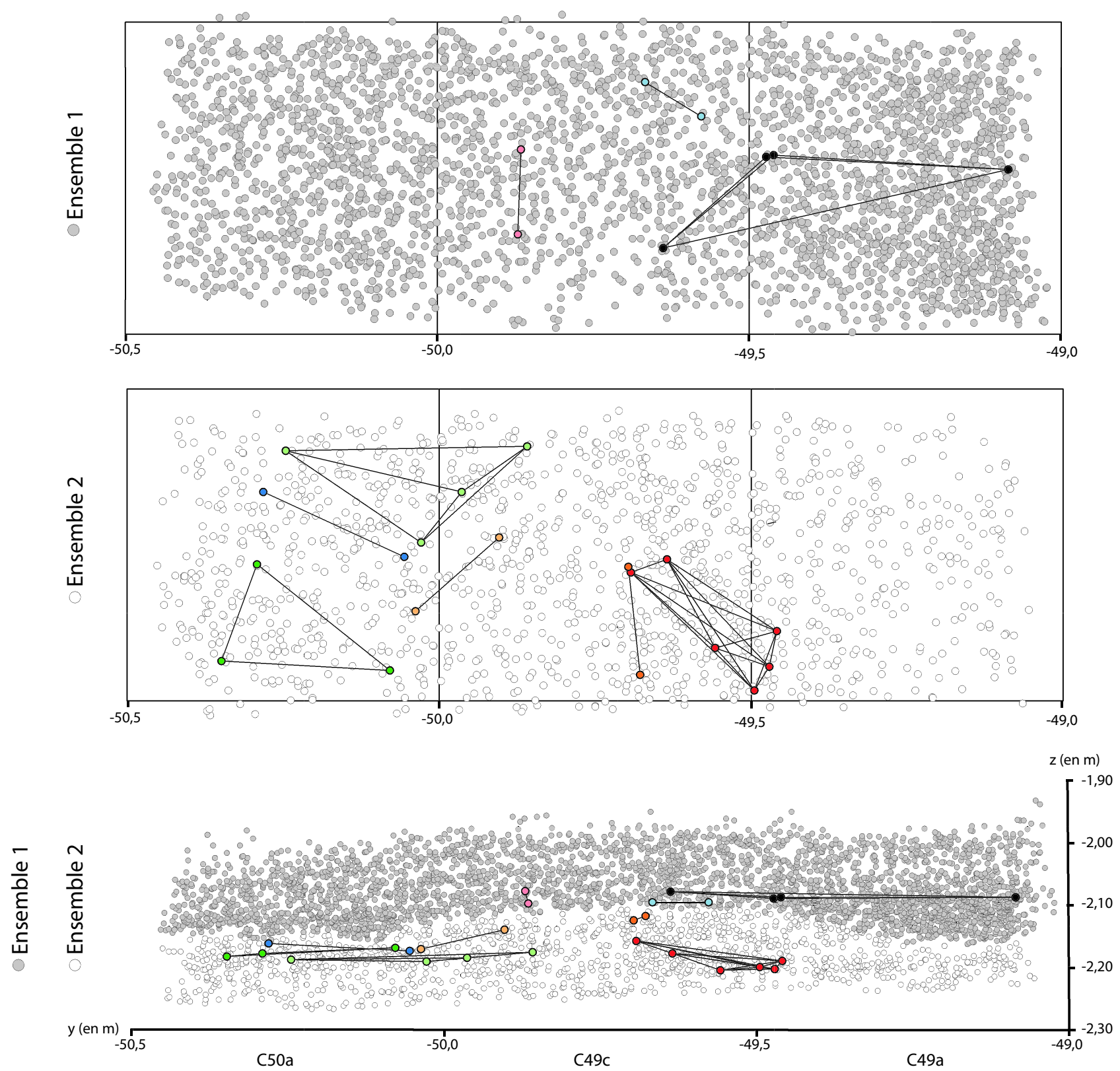

- FIGURE 14 -

Projections en plan et en coupe sagittale des liens des raccords effectués au sein

Plan and sagittal projections of refits within ensembles 1 and 2. des ensembles 1 et 2

majorité une forme moins aplatie les rendant ainsi plus facilement mobilisables par les écoulements d'eau. Ce constat est d'autant plus vrai pour les vestiges fauniques brûlés, plus fragmentés et pouvant perdre jusqu'à $30 \%$ de leur masse au cours de leur combustion (Zazzo et al. 2009). Les résultats de la granulométrie des vestiges lithiques laissent donc entrevoir une redistribution et une recomposition des ensembles potentiellement plus conséquentes pour les vestiges fauniques, moins denses et donc plus mobiles lors d'écoulements d'eau.

Cette hypothèse est appuyée par les analyses de fabriques qui mettent en évidence pour certains échantillons de mesures de vestiges fauniques des données comparables à celles obtenues lors d'expériences de ruissellement réalisées en milieu naturel. La nappe de vestiges fauniques brûlés observée sur les projections dans l'ensemble 1 pourrait s'expliquer par des écoulements d'eau lors d'un épisode de crue dont la compétence très faible aurait suffit à éroder en amont du site les particules les plus fines et les moins denses -soit les vestiges lithiques de 2 à $4 \mathrm{~mm}$, les vestiges fauniques brûlés et les vestiges fauniques les plus mobiles- qu'ils auraient redéposé dans la zone fouillée actuellement. Lors de cette remobilisation, des tris ont pu intervenir selon la structure du vestige faunique (spongieux versus compact), l'élément anatomique (Dominguez-Rodrigo et al. 2018), voire, selon le stade de combustion du reste. 
$\mathrm{n}=35$

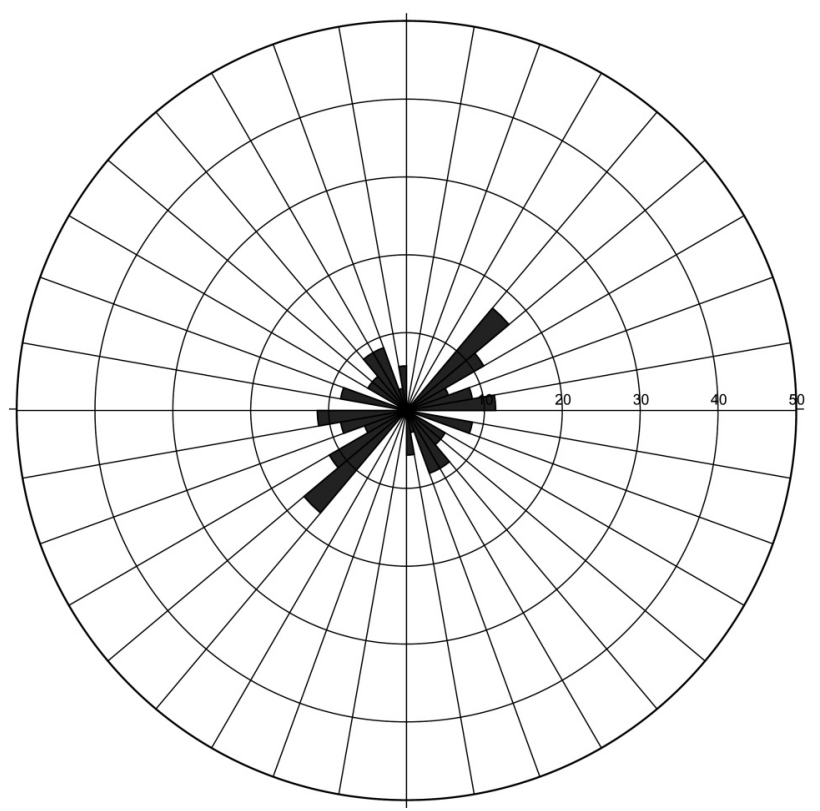

(3) Les résultats de l'analyse statistique des états de surface du matériel lithique révèlent des phénomènes intéressants mais ne permettent pas d'individualiser les deux ensembles étudiés

Le piétinement humain est susceptible de redistribuer horizontalement et verticalement les vestiges (Hughes et Lampert 1977 ; Villa 1982 ; Villa et Courtin 1983) mais également d'altérer le bord des pièces lithiques (Bordes 1961 ; Prost 1989 ; McBrearty 1998 ; Thiébaut et al. 2010 ; McPherron et al. 2014) ou encore, de fragmenter les os, et plus encore, les os brûlés (Thiébaut et al. 2010). Lors d'une expérience sur les conséquences du piétinement, S. McPherron et collaborateurs (2014) démontrent que les enlèvements apparaissent sur la face opposée à celle reposant sur le sol et qu'ils ne connaissent pas une répartition aléatoire sur les éclats. D'une part, ils atteignent plus facilement les bords les plus fragiles, soit, sur un éclat, les tranchants aigus. D'autre part, ils se manifestent de manière inégale selon la disposition de la pièce : les pièces qui reposent sur leur face inférieure tendent à acquérir plus d'enlèvements mécaniques que les pièces reposant sur leur face supérieure. Sans contrôler la face de repos des pièces dans ces expériences, D.-C. Prost constate également une plus forte proportion d'altérations sur la face supérieure des pièces (Prost 1989). Une surface de contact plus importante avec le sol lorsque l'éclat repose sur la face inférieure pourrait expliquer ce phénomène. Nos résultats mettent également en avant une très forte proportion d'altérations mécaniques sur la face supérieure des pièces. Le rôle des écoulements d'eau pourrait expliquer ce phénomène puisqu'il a pu positionner les objets sur leur face de plus forte stabilité, soit la face inférieure des éclats contre le sol (Schick 1986). Malheureusement, la face de repos des objets n'a pas été relevée à la fouille et cette hypothèse ne peut être discutée de manière plus précise. De plus, nous pensons que le rôle des écoulements d'eau qu'il soit d'origine fluviatile ou lié au ruissellement est secondaire dans l'altération mécanique du bord des pièces (cf. les résultats de la granulométrie lithique et des analyses de fabriques).

\section{FIGURE 15}

Diagramme en rose de l'orientation des liens de raccords réalisés sur les vestiges lithiques de l'ensemble 2.

Rose diagram ofrefit orientations for lithic artefacts from ensemble 2.

Le piétinement humain au grès de nombreuses occupations et réoccupations du site est, selon ces résultats, le principal facteur explicatif de l'altération mécanique du bord des vestiges. Nous pensons que le piétinement a également causé (au moins pour partie) la fragmentation des vestiges fauniques brûlés, dans l'ensemble 2 notamment. Dans ce dernier ensemble, un taux de sédimentation lent a laissé les vestiges archéologiques exposés sur un laps de temps plus long et ainsi, de manière répétée au piétinement humain lors des phases d'occupation du site, provoquant l'altération mécanique du bord des vestiges lithiques et une probable fragmentation des vestiges fauniques brûlés.

L'association des états de surface ne permet pas de distinguer les deux ensembles étudiés et met en évidence une forte homogénéité de l'assemblage quels que soient le concept ou la méthode de taille.

\section{(4) Quels scénarios de réorganisation spatiale et de recomposition des nappes de vestiges originelles pour les ensembles 1 et 3 ?}

Les résultats obtenus pour l'ensemble 2 appuient l'étude géo-archéologique qui met en évidence des processus dépositionnels et post-dépositionnels favorisant une bonne conservation de la composition et de l'organisation originelle des nappes de vestiges. En revanche, les outils analytiques exposés ci-dessus ne permettent pas de soumettre de conclusions sûres concernant la formation des ensembles 1 et 3 , mais de premières hypothèses peuvent être émises.

La formation et la composition des nappes de vestiges des ensembles 1 et 3 dépend d'une part des lignes de courant lors de la crue et de leurs compétences (Texier op. cit.) et, d'autre part de la prédisposition à la mobilité des vestiges (i.e. propriétés physiques intrinsèques du vestige : forme, taille, densité du matériau) face aux écoulements d'eau. La crue responsable de la réorganisation des nappes de vestiges formant l'ensemble 1 a probablement tout d'abord accumulé des objets lithiques dans des dépressions au sommet de l'éboulis que formait l'ensem- 
ble 2, créant ainsi des nappes de vestiges très denses. Par dessus, la compétence des écoulements d'eau s'amenuisant, il est probable que ce soit alors déposé une nappe de vestiges composées des éléments les plus mobiles en contexte fluviatile, dont des restes fauniques brûlés probablement érodés en amont du site. L'absence de faune brûlée dans l'ensemble 3 pourrait s'expliquer par des phénomènes de tri inverses à ceux constatés dans l'ensemble 1, c'est-à-dire, une érosion de ce type de vestige ou bien, à une différence de fonctionnement de cette partie du site (par exemple, l'absence de structures de combustion à cette époque dans cette partie du site).

\section{6 | CONCLUSION}

Les différences de densité et de proportions entre matériaux à l'abri inférieur du Moustier peut s'expliquer par le biais de plusieurs facteurs

(1) La place prise par les blocs calcaires lors des épisodes d'effondrement des parois de l'abri donne une impression de moindre densité sur le terrain et sur les projections du matériel coté. Cette impression est confirmée par la mesure de densité stricte de matériel par litre de sédiment. Ainsi, l'ensemble 2 est bel et bien moins dense que les ensembles 1 et 3 , indépendamment de la fréquence des blocs calcaires.

(2) Le piétinement humain, reconnu grâce à l'analyse de l'altération mécanique du bord des vestiges lithiques est probablement responsable de la fragmentation d'une partie des vestiges fauniques brûlés. On peut s'attendre à ce que cette fragmentation est d'autant plus importante que la sédimentation est lente et ainsi, que la période d'exposition de l'objet à l'action du piétinement et au weathering est prolongée. Cependant, ces facteurs ne peuvent expliquer à eux seuls la relative rareté des restes de faunes brûlés dans certains niveaux.

(3) Des phénomènes de tri inter-matériaux selon la prédisposition à la mobilité du vestige face aux écoulements d'eau sont fortement suspectés dans les ensembles réorganisés et scellés lors des épisodes de crue de la Vézère (ensembles 1 et 3). Ainsi, l'organisation et la composition finale des nappes de vestiges des ensembles 1 et 3 sont dépendantes de phénomènes naturels, parmi lesquels figurent les processus fluviatiles dont il s'agit de mesurer l'impact sur l'intégrité des ensembles archéologiques.

Dans le cas de la zone B de l'abri inférieur du Moustier, les chaînes opératoires décrites par l'analyse technoéconomique (Thomas et Gravina, ce volume) sont homogènes et indiquent une persistance des choix techniques et économiques. Les résultats de l'analyse taphonomique et spatiale révèlent une forte homogénéité de ces ensembles et légitiment ainsi la réattribution de ces industries au techno-complexe Discoïde (Gravina et Discamps 2015). Pour l'heure, en l'absence de datations plus précises pour les périodes anciennes, la densité des nappes de vestiges est difficilement interprétable. Ces densités disparates, sont le fait de l'interaction entre des facteurs anthropiques (modalités d'occupation du site, durée, fréquence, fonction) et des facteurs naturels (vitesse de sédimentation, agents post-dépositionnels) dont il s'agit de mesurer et de discuter les effets. À l'abri inférieur du Moustier, l'interaction entre les activités menées par les néandertaliens sur la faune (combustion ou nettoyage du camp) d'une part et, les processus de sédimentation (processus fluviatiles, ruissellement, éboulisation) et certains agents taphonomiques (piétinement) d'autre part, expliquent les différences de densités observées à la fouille.

\section{REMERCIEMENTS}

Nous remercions vivement Jean-Jacques Cleyet-Merle pour nous avoir autorisé l'accès aux collections du Moustier stockées au Musée National de la Préhistoire des Eyzies et l'ensemble des personnels de ce musée pour leur aide si précieuse (et tout particulièrement Stéphane Madelaine, Peggy Jacquement et Bernard Nicolas). Une partie des analyses rapportées ici a été réalisée dans le cadre d'un travail de Master à l'Université Toulouse Jean Jaurès et au sein de l'UMR5608 TRACES sous la direction de E. Discamps et B. Gravina. Ainsi, nous tenons à remercier l'équipe pédagogique de ce Master pour son soutien, également J.-G. Bordes et F. Bon alors relecteurs de ce travail.

\section{RÉFÉRENCES BIBLIOGRAPHIQUES}

BERTRAN P., CLAUD É., DETRAIN L., LENOBLE A., MASSON B., VALLIN L. 2006 - Composition granulométrique des assemblages lithiques, application à l'étude taphonomique des sites paléolithiques, Paleo, $n^{\circ}$ 18, p. 7-36.

BERTRAN P., LENOBLE A. 2002 - Fabriques des niveaux archéologiques: méthode et premier bilan des apports à l'étude taphonomique des sites paléolithiques, Paleo, $n^{\circ} 14,19 p$

BERTRANP., LENOBLE A., TODICSO D., DESROSIERS P., SORENSEN M. 2012 - Practicle size distribution of lithic assemblages and taphonomy of Palaeolithics sites, Journal of Archaeological Science, n 39 (10), p. 3148-3166.

BORDES F. 1948 - Les couches moustériennes du gisement du Moustier (Dordogne). Typologie et techniques de taille, Bulletin de la Société Préhistorique Française, tome 45, $n^{\circ} 4-5$, p. 113-124.

BORDES F. 1961 - Typologie du Paléolithique ancien et moyen, Delmas, Publications de l'Institut de Préhistoire de l'Université de Bordeaux, Mémoire $n^{\circ} 1$, Bordeaux, 111 p.

BORDES J.-G. 2002 - Les interstratifications Chatelperronien/Aurignacien du Roc-de-Combe et du Piage (Lot, France). Analyse taphonomique des industries lithiques; implications archéologiques, Université de Bordeaux, Thèse de doctorat, $423 \mathrm{p}$.

BORDES J.-G. 2012 - À propos de taphonomie lithique : fondements épistémologiques et outils analytiques. Réflexions à partir du thème de la transition du Paléolithique moyen au Paléolithique supérieur, habilitation à diriger des recherches, Université de Bordeaux I, Bordeaux, $114 \mathrm{p}$.

BRAVARD J.-P., PETIT F. 1997 - Les cours d'eau, dynamique du système fluvial, Paris, Armand Colin, Collection U, 222 p. 
BRUGAL J.-P. 2006 - Petit gibier et fonction de sites au Paléolithique supérieur, Paleo, nº18, p. 45-68.

CAUX S., GALLAND A., QUEFFELEC A., BORDES J.-G. 2018 Aspect and characterization of chert alteration in an archaeological context: A qualitative to quantitative pilot study, Journal of Archaeological science: Reports, $n^{\circ} 20$, p. 210-2019.

DISCAMPS E., BACHELLERIE F., BAILLET M., SITZIA L. 2019 - The use of spatial taphonomy for interpretating Pleistocene palimpstests : an interdisciplinary approach to the Châtelperronian and carnivore occupations at Cassenade (Dordogne, France), PaleoAnthropology, p. 362-388

DISCAMPS E., LEMEUR C. 2019 - Variabilité des proies chassées et modalités d'exploitation du Cerf au Moustérien: l'apport des collections récentes du Moustier (couches $\mathrm{G}$ et $\mathrm{H})$, Paleo, $n^{\circ}$ 30, tome 1, p. 318-330.

DISCAMPS E., MUTH X., GRAVINA B., LACRAMPES-CUYAUBERES F., CHADELLE J.-P., FAIVRE J.-P., MAUREILLE B. 2016 Photogrammetry as a tool for interpreting archival data in archaeological fieldwork: Examples from the Middle Plaeolithic sites of Combe-Grenal, Le Moustier and Regourdou, Journal of Archaeological science: Reports, volume 8 , p. 268-276

DOEGLAS D.J. 1962 - The structure of sedimentary deposits of braided rivers, Sedimentology 1(3), p. 167-190.

DOMINGUEZ-RODRIGO M., COBO-SANCHEZ L., YRAVEDRA J., URRIBELAEREA D., ARRIAZA C., ORGANISTA E., BAQUEDANO E. 2018 - Fluvial spatial taphonomy: a new method for the study of post depositional processes, Archaeology Anthropology Science 10, p. 1769-1789.

FERNANDES P. 2012 - Itinéraires et transformations du silex: une pétroarchéologie refondée, application au Paléolithique moyen, thèse de doctorat, Université de Bordeaux I, Bordeaux, 623 p.

GRAVINA B. 2017 - Intra-level technological change and its implications for Mousterian assemblage variability. The example of Le Moustier, layer G. Quaternary International 433, p. 132-139.

GRAVINA B., DISCAMPS E. 2015 - MTA-B or not to be ? Recycled bifaces and shifting hunting strategies at Le Moustier and their implication for the late Middle Palaeolithic in southwestern France. Journal of Human Evolution 84, p. 83-98

GRAVINA B., DISCAMPS E., D'ERRICO, GUERIN G., LAYHAYE C., PITARCH A., ROYER A., TEXIER J.-P., THOMAS M. 2018 - Le Moustier (Saint-Léon sur Vézère), Rapport de fouille, Bordeaux, Service Régional de l'Archéologie, 103 p.

GRAVINA B., DISCAMPS E., GUERIN G., LAHAYE C., LEMEUR C., PITARCH A., TEXIER J.-P., THOMAS M. 2016 - Le Moustier (Saint-Léon sur Vézère), Rapport de fouille, Bordeaux, Service Régional de l'Archéologie, 95 p.

GRAVINA B., DISCAMPS E., GUERIN G., LAHAYE C., LEMEUR C., PITARCH A., TEXIER J.-P., THOMAS M. 2017 - Le Moustier (Saint-Léon sur Vézère), Rapport de fouille, Bordeaux, Service Régional de l'Archéologie, 102 p.
GRAVINA B., DISCAMPS E., TEXIER J.-P., FAURE J., GUERIN G., LAHAYE C., ROYER A. 2015 - Le Moustier (Saint-Léon sur Vézère), Rapport de fouille, Bordeaux, Service Régional de l’Archéologie, 92 p.

GRAVINA B., LAHAYE C., DISCAMPS E., ROYER A., TEXIER J.-P., GUERIN G. 2014 - Le Moustier (Saint-Léon sur Vézère), Rapport de fouille, Bordeaux, Service Régional de l'Archéologie, 74 p.

HISCOCK P. 1981 - Comments on the use of chipped stone artefacts as a measure of "intensity site usage », Australian archaeology, $n^{\circ} 13$, p. 30-34

HUGHES P.J., LAMPERT R. J. 1977 - Occupational disturbance and type of archaeological deposits, Journal of Archaeological Science, $n^{\circ}$ 4, p. 135-140.

ISAAC G. 1967 - Toward the interpretation of occupation debris: some experiments and observations, Kroeber Anthopological Society Papers, $n^{\circ} 37$, p. 31-57

LARTET E., CHRISTY H. 1865-1875 - Reliquiae Aquitanicae, being contributions to the archaeology and Palaeontology of Perigord and the adjoining provinces of Southern France, Londres, $506 \mathrm{p}$.

LAVILLE H., RIGAUD J.-P. 1973 - L'abri inférieur du Moustier (Dordogne). Précisions stratigraphiques et chronologiques. Compte Rendu de l'Académie des Sciences de Paris. Tome 276, p. 3097-3100.

LE S., JOSSE J. HUSSON F. 2008 - FactoMineR: An R Package for Multivariate Analysis. Journal of Statistical Software, 25(1), p. 1-18.

LENOBLE A. 2005 - Ruissellement et formation des sites préhistoriques : référentiel actualiste et exemples d'application au fossile. Oxford, British Archaeological Report International Series, $n^{\circ}$ 1363, 212 p.

LENOBLE A., TEXIER J.-P. 2016 - Processus géologiques de formation du site d'Isturitz (Sud-Ouest de la France), Paleo, $n^{\circ} 27$, p. 235-252.

MCBREARTY S., BISHOP L., PLUMMER T., DEWAR R., CONARD N. 1998 - Tools underfoot: human trampling as an agent of lithic artifact edge modification. American Antiquity, $n^{\circ}$ 63/1, p. 108-129.

MCPHERRON S. P., BRAUN D. R., DOGANDZIC T., ARCHER W., DESTA D., LIN S. C. 2014 - An experimental assessment of the influences on edge damage to lithic artifacts : a consideration of edge angle, substrate grain size, raw material properties, and exposed face, Journal of Archaeological Science, $n^{\circ} 49$, p. $70-82$

MIALL A. D. 1996 - The geology of fluvial deposits. Springer ed., $582 \mathrm{p}$.

MORTILLET G. (de) 1869 - Essai d'une classification des cavernes et des stations sous abri fondée sur les produits de l'industrie humaine, Typographie de Bonnal et Gibrac, $8 \mathrm{p}$. 
PARGETER J., LOFTUS E., MACKAY A., MITCHELL P., STEWART B. 2018 - New Ages from Boomplaas Cave, South Africa, provide increase resolution on late/terminal Pleistocene human behavioural variability, Archaeological Research in Africa, volume 53, p. 156-184

PEYRONY D. 1930 - Le Moustier. Ses gisements, ses industries, ses couches géologiques. Revue Anthropologique, tome XL, p. 48-76 et p. 155-176.

PROST D.-C. 1989 - Enlèvements accidentels, enlèvements d'utilisation et de retouche sur les outils de pierre taillée, Université de Paris X - Nanterre, thèse de doctorat, 552 p.

REINECK H.-E., SINGH I.-B. 1980 - Depositional sedimentary environments, Springer-Verlag, New-York, 549 p.

RENDU W., BOURGUIGNON L., COSTAMAGNO S., MEIGNEN L., SOULIER M.-C., ARMAND D., BEAUVAL C., DAVID F., GRIGGO C., JAUBERT J., MAUREILLE B., PARK S.-J. 2011 - Approche interdisciplinaire et réflexions méthodologiques sur la question des haltes de chasse au Moustérien, in Bon Fr., Costamagno S., Valdeyron N. (dir.), Haltes de chasse en Préhistoire. Quelles réalités archéologiques?, Actes du colloque international du 13 au 15 mai 2009, Université Toulouse II - Le Mirail, P@lethnologie, n³, p. 61-75.

SCHICK K.D. 1986 - Sites in the Making, Experiments in the Formation and Transformation of Archaeological Occurrences, Oxford, BAR International Series 319, 313 p.

SCHIFFER M.B. 1983 -Toward the identification of Formation Processes, Society for American Archaeology, vol. 48, $n^{\circ}$, p. 675-706

SORESSI M. 2002 - Le Moustérien de tradition acheuléenne du sud-ouest de la France, Discussion sur la signification des faciès à partir de l'étude comparée de 4 sites: Pech de l'Azé I, le Moustier, La Rochette et la Grotte XVI, thèse de doctorat, Université de Bordeaux I, Bordeaux, 344 p.

TEXIER P.-J. 1981 - Dessilicification des silex taillés, Quaternaria Nova 23, p. 159-169

TEXIER J.-P. 2000 - À propos des processus de formation des sites préhistoriques. Paleo, n 12 , p. 379-386.
TEXIER J.-P. 2009 - Histoire géologique de sites préhistoriques classiques du Périgord : une vision actualisée : la Micoque, la grotte Vaufrey, le Pech de l'Azé I et II, la Ferrassie, l'abri Castanet, le Flageolet, Laugerie Haute, Paris, Éditions du Comité des travaux historiques et scientifiques, p. 196.

THIÉBAUT C., COSTAMAGNO S., COUMONT M.-P., MOURRE V., PROVENZANO N., THÉRY-PARISOT I. 2010 - Approche expérimentale des conséquences du piétinement des grands herbivores sur les vestiges lithiques et osseux, In : C. Thiébaut, M.-P. Coumont, A. Averbouh, (éd.), Actes du workshop n 16 $X V$ e congrès international de l'UISPP Lisbonne, septembre 2006, Paleo - supplément n³, p. 109-130.

THOMAS M. 2017 - Taphonomie et techno-économie de palimpsestes d'occupations : l'exemple de la couche $\mathrm{H} d u$ Moustier (Saint-Léon sur Vézère, Dordogne), Université Toulouse Jean Jaurès, Mémoire de Master 2, 69 p.

THOMAS M., GRAVINA B. 2019 - Analyse techno-économique des vestiges lithiques de la zone $B$ de l'abri inférieur du Moustier (Dordogne, France), Paleo, n 30 , tome 1, p. 300-317.

VALLADAS H., GENESTE J.-M., JORON J.-M., CHADELLE J.-P. 1986 - Thermoluminescence dating of the Moustier (Dordogne, France), Nature, $n^{\circ} 322$, p. 452-454.

VILLA P. 1982 - Conjoignable pieces and site formation processes. American Antiquity, nº 47, p. 276-290.

VILLA P., COURTIN J. 1983 - The Interpretation of Stratified Sites: A View from Underground. Journal of Archaeological Science, $n^{\circ} 10$, p. 267-281.

WEAVER T.D., BOYCO R.H., STEELE T.E. 2011 - Cross-platform program for likelihood-based statistical comparisons of mortality profiles on a triangular graph. Journal of Archaeological Science 38, p. 2420-2423.

ZAZZO A., SALIĖGE J.-F., PERSON A., BOUCHER H. 2009 Radiocarbon dating of calcined bones: where does the carbon come from ?, Radiocarbon, vol. 51, n² 2, p. 601-611. 